\title{
APPROXIMATION OF ROUGH PATHS OF FRACTIONAL BROWNIAN MOTION
}

\author{
ANNIE MILLET ${ }^{*, \dagger}$ AND MARTA SANZ-SOLÉ *
}

\begin{abstract}
We consider a geometric rough path associated with a fractional Brownian motion with Hurst parameter $H \in] \frac{1}{4}, \frac{1}{2}[$. We give an approximation result in a modulus type distance, up to the second order, by means of a sequence of rough paths lying above elements of the reproducing kernel Hilbert space.
\end{abstract}

\section{INTRODUCTION}

Consider a $d$-dimensional fractional Brownian motion $W^{H}$ with Hurst parameter $H \in] \frac{1}{4}, \frac{1}{2}[\cup] \frac{1}{2}, 1[$ and integral representation

$$
W_{t}^{H}=\int_{0}^{1} K^{H}(t, s) d B_{s},
$$

where $K^{H}(t, s)=0$, if $s \geq t$ and for $0<s<t$,

$$
K^{H}(t, s)=c_{H}(t-s)^{H-\frac{1}{2}}+s^{H-\frac{1}{2}} F_{1}\left(\frac{t}{s}\right)
$$

with

$$
F_{1}(z)=c_{H}\left(\frac{1}{2}-H\right) \int_{0}^{z-1} u^{H-\frac{3}{2}}\left(1-(u+1)^{H-\frac{1}{2}}\right) d u
$$

for $z>1$ (see for instance [1], equation (42)). In (1.1), $B$ denotes a standard $d$-dimensional Brownian motion and in (1.2), (1.3), $c_{H}$ denotes a positive real constant depending on $H$.

Let $p \in] 1,4[$ be such that $p H>1$. In [2], it is proved that the sequence of smooth rough paths based on linear interpolations of $W^{H}$ converges in the $p$ variation distance. The limit defines a geometric rough path with roughness $p$ lying above $W^{H}$. We will call this object the enhanced fractional Brownian motion.

1991 Mathematics Subject Classification. MSC 2000: Primary 60G15, Secondary $60 \mathrm{H} 05,60 \mathrm{H} 07$.

Key words and phrases. Fractional Brownian motion, rough paths.

* Supported by the grants BFM 2003-01345, HF 2003-006, Dirección General de Investigación, Ministerio de Educación y Ciencia, Spain.

$\nmid$ Partially supported by the program SAB 2003-0082, Dirección General de Universidades, Ministerio de Educación y Ciencia, Spain. 
In the recent papers [5], 3], the $p$-variation distance on rough paths is replaced by a strictly stronger, modulus type distance defined as follows:

$$
\bar{d}_{p}(x, y)=\sup _{0 \leq s<t \leq 1}\left(\sum_{i=1}^{[p]} \frac{\left|x_{s, t}^{(i)}-y_{s, t}^{(i)}\right|}{(t-s)^{\frac{i}{p}}}\right) .
$$

In [3], it is proved that the enhanced fractional Brownian motion can actually be obtained by means of the $\bar{d}_{p}$ distance and also that linear interpolations of $W^{H}$ define stochastic processes with values in $\mathcal{H}^{H}$, the reproducing kernel Hilbert space associated with $W^{H}$ (see Theorem 3.3 in [4 for a description of this space). Then, the authors state a characterization of the topological support of the enhanced fractional Brownian motion among other results.

Our aim in this work is to give a new approximation of the enhanced fractional Brownian motion by means of a sequence of geometric rough paths which, unlike those based on linear interpolations, are not smooth, but also belong to $\mathcal{H}^{H}$. For the sake of simplicity, we restrict to $[p]=2$. We are pretty confident that our results extend to $[p]=3$; however, dealing with higher generality would most likely produce a very technical paper. Our result, as is stated in Theorem 2.1 provides in particular a new approximation of the Lévy area of the fractional Brownian motion.

For any $m \in \mathbb{N}$, we consider the dyadic grid $\left(t_{k}^{m}=k 2^{-m}, k=0,1, \ldots, 2^{m}\right)$ and set $\left.\left.\Delta_{k}^{m}=\right] t_{k-1}^{m}, t_{k}^{m}\right]$ and $\Delta_{k}^{m} B=B_{t_{k}^{m}}-B_{t_{k-1}^{m}}$. Define $B(m)_{0}=0$ and for $t \in \Delta_{k}^{m}, B(m)_{t}=B_{t_{k-1}^{m}}+2^{m}\left(t-t_{k-1}^{m}\right) \Delta_{k}^{m} B$. Our approximation sequence is defined by

$$
W(m)_{t}^{H}=\int_{0}^{t} K^{H}(t, s) \dot{B}(m)_{s} d s
$$

$m \in \mathbb{N}$, where $\dot{B}(m)_{s}$ denotes the derivative with respect to $s$ of the path $s \mapsto B(m)_{s}$. Notice that $W(m)^{H} \in \mathcal{H}^{H}$.

Let $K_{m}^{H}$ be the orthogonal projection of $K^{H}(t, \cdot)$ on the $\sigma$-field generated by $\left(\Delta_{k}^{m}, k=1, \cdots, m\right)$. That is, for any $0<s<t \leq 1$,

$$
K_{m}^{H}(t, s)=\sum_{k=1}^{2^{m}} 2^{m}\left(\int_{\left.\left.\Delta_{k}^{m} \cap\right] 0, t\right]} K^{H}(t, u) d u\right) \mathbf{1}_{\Delta_{k}^{m}}(s) .
$$

We clearly have

$$
W(m)_{t}^{H}=\int_{0}^{1} K_{m}^{H}(t, s) d B_{s} .
$$

For $H \in] \frac{1}{2}, 1\left[\right.$, we set $\mathbf{W}=\left(\mathbf{W}_{s, t}=\left(W_{s, t}^{(1)}, 0 \leq s \leq t \leq 1\right), \mathbf{W}(\mathbf{m})=\right.$ $\left(\mathbf{W}(\mathbf{m})_{s, t}=\left(W(m)_{s, t}^{(1)}, 0 \leq s \leq t \leq 1\right)\right.$, while for $\left.H \in\right] \frac{1}{4}, \frac{1}{2}[$ we set $\mathbf{W}=$ $\left(\mathbf{W}_{s, t}=\quad\left(W_{s, t}^{(1)}, W_{s, t}^{(2)}\right.\right.$, $0 \leq s \leq t \leq 1)$ and $\mathbf{W}(\mathbf{m})=\left(\mathbf{W}(\mathbf{m})_{s, t}=\left(W(m)_{s, t}^{(1)}, W(m)_{s, t}^{(2)}, 0 \leq s \leq\right.\right.$ $t \leq 1), m \geq 1$. 
The main result of the paper states the convergence of $\mathbf{W}(\mathbf{m})$ to $\mathbf{W}$ in the $\bar{d}_{p}-$ metric for $\left.p \in\right] 1,3[$. For $p \in] 1,2[$, the result is an almost trivial consequence of Lemma 3.2 which establishes Hölder continuity in the $L^{2}[0,1]$ norm of the kernels $K^{H}, K_{m}^{H}$, respectively, and a control of the quadratic mean error in the approximation of $K^{H}$ by $K_{m}^{H}$. For $p \in[2,3$ [, the approximation of the Lévy area relies on representation formulas for the second order multiple integrals by means of the operator $K^{*}$ given in (2.3) and introduced in [1] (see also [2]). There are two fundamental ingredients. Firstly, Proposition [2.3, giving the rate of convergence of the approximation at the second order level in the $L^{q}(\Omega)$-modulus norm; secondly, Lemma 3.5, an extension of the Garsia-Rademich-Rumsey Lemma for geometric rough paths of any roughness $p$. Other technical results used in the proofs, mainly on the kernels $K^{H}$ and $K_{m}^{H}$, are given in the Appendix.

For simplicity, in general we shall not write explicitly the dependence on $H$; thus $W$ stands for $W^{H}, K(t, s)$ for $K^{H}(t, s)$, etc. For any $q \in[1, \infty[$, we denote by $\|\cdot\|_{q}$ the $L^{q}(\Omega)$-norm. We make the convention $\sum_{k=a}^{b} x_{k}=0$ if $b<a$ and denote by $C$ positive constants with possibly different values. For additional notions and notation on rough paths, we refer the reader to [ 6 .

\section{Approximation Result}

For $p \in] 1,+\infty\left[\right.$ we set $\tilde{d}_{p}=\bar{d}_{p \wedge 2}$, that is

$$
\tilde{d}_{p}(x, y)=\sup _{0 \leq s<t \leq 1}\left(\sum_{i=1}^{[p] \wedge 2} \frac{\left|x_{s, t}^{(i)}-y_{s, t}^{(i)}\right|}{(t-s)^{\frac{i}{p}}}\right) .
$$

The purpose of this section is to prove the following approximation result.

Theorem 2.1. Let $H \in] \frac{1}{4}, \frac{1}{2}[, p \in] 2,4[$ (resp. $H \in] \frac{1}{2}, 1[, p \in] 1,2[$ ), be such that $p H>1$ and $q \in\left[1,+\infty\left[\right.\right.$. The sequence $\left(\tilde{d}_{p}(\mathbf{W}(\mathbf{m}), \mathbf{W}), m \geq\right.$ $1)$, converges to 0 in $L^{q}(\Omega)$ and a.s. Thus for $\left.H \in\right] \frac{1}{2}, 1[$ and $p \in] 1,2[$, if $\mathcal{G}_{p}$ denotes the set of dyadic geometric rough paths endowed with the norm $\tilde{d}_{p}(0,$.$) and P^{H}$ denotes the law of the fractional Brownian motion $W^{H}$, then the triple $\left(X, \mathcal{H}^{H}, P^{H}\right)$ is an abstract Wiener space.

The next Proposition provides the auxiliary result to state the approximation of the first component of the enhanced fractional Brownian motion.

Proposition 2.2. Let $0 \leq s<t \leq 1, q \in[1, \infty[$.

(i) For any $H \in] 0, \frac{1}{2}[, \lambda \in[0, H[$,

$$
\left\|W_{s, t}^{(1)}-W(m)_{s, t}^{(1)}\right\|_{q} \leq C 2^{-m \lambda}|t-s|^{H-\lambda} \text {. }
$$

(ii) For any $H \in] \frac{1}{2}, 1\left[, \varepsilon \in\left[0, H[, \mu \in] 0, \frac{\varepsilon}{H(2 H+1)}[\right.\right.$,

$$
\left\|W_{s, t}^{(1)}-W(m)_{s, t}^{(1)}\right\|_{q} \leq C 2^{-m \mu}|t-s|^{H-\varepsilon} .
$$


Proof. By the hypercontractivity inequality, it suffices to prove the results for $q=2$. In this case, it is an easy consequence of the identity

$E\left(\left|W_{s, t}^{(1)}-W(m)_{s, t}^{(1)}\right|^{2}\right)=\int_{0}^{1}\left|(K(t, u)-K(s, u))-\left(K_{m}(t, u)-K_{m}(s, u)\right)\right|^{2} d u$ and of Lemma 3.2. Indeed, by (3.14), we have

$$
E\left(\left|W_{s, t}^{(1)}-W(m)_{s, t}^{(1)}\right|^{2}\right) \leq C|t-s|^{2 H} .
$$

Hence, if $t-s<2^{-m}$, we easily obtain (2.1) and (2.2).

Assume now $H \in] 0, \frac{1}{2}\left[\right.$ and $t-s \geq 2^{-m}$. By (3.15), for $\epsilon \in[0, H]$,

$$
E\left(\left|W_{s, t}^{(1)}-W(m)_{s, t}^{(1)}\right|^{2}\right) \leq C 2^{-2 m H} \leq C 2^{-2 m \epsilon}|t-s|^{2(H-\epsilon)} .
$$

Hence, (2.1) follows.

Let $H \in] \frac{1}{2}, 1\left[\right.$ and $t-s \geq 2^{-m}$. Let $\left.\alpha \in\right] 0,1[$; then (3.14) and (3.16) imply

$$
\left\|W_{s, t}^{(1)}-W(m)_{s, t}^{(1)}\right\|_{q} \leq C|t-s|^{H(1-\alpha)} 2^{-m \lambda \alpha},
$$

with $\lambda \in] 0, \frac{1}{2 H+1}\left[\right.$. By taking $\alpha=\frac{\varepsilon}{H}$, we obtain (2.2) with $\mu=\lambda \frac{\varepsilon}{H}$.

Throughout the rest of this section, $H \in] \frac{1}{4}, \frac{1}{2}\left[\right.$. Following [1], let $\mathcal{H}_{K}$ denote the set of functions $\varphi:[0,1] \rightarrow \mathbb{R}$ such that

$$
\|\varphi\|_{K}^{2}=\int_{0}^{1} \varphi(s)^{2} K(1, s)^{2} d s+\int_{0}^{1} d s\left(\int_{s}^{1}|\varphi(t)-\varphi(s)||K|(d t, s)\right)^{2}<+\infty .
$$

For any $\varphi \in \mathcal{H}_{K}, 0<s<t$, set

$$
\begin{aligned}
& K^{*}\left(\mathbf{1}_{] s, t]}(\cdot)\left(\varphi .-\varphi_{s}\right)\right)(u)=\mathbf{1}_{] 0, s]}(u) \int_{s}^{t}\left(\varphi_{r}-\varphi_{s}\right) K(d r, u) \\
& \quad+\mathbf{1}_{s, t]}(u)\left(K(t, u)\left(\varphi_{u}-\varphi_{s}\right)+\int_{u}^{t}\left(\varphi_{r}-\varphi_{u}\right) K(d r, u)\right) .
\end{aligned}
$$

Following [2],

$$
W_{s, t}^{(2)}=\int_{0}^{1} K^{*}\left(\mathbf{1}_{] s, t]}(\cdot)\left(W .-W_{s}\right)\right)(u) d B_{u}+\frac{1}{2}|t-s|^{2 H} .
$$

Moreover, by Theorem 9 in [7, for $W(m)$ defined in (1.4) we have

$$
W(m)_{s, t}^{(2)}=\int_{0}^{1} K^{*}\left(\mathbf{1}_{] s, t]}(\cdot)\left(W(m) .-W(m)_{s}\right)\right)(u) \dot{B}(m)_{u} d u .
$$

Proposition 2.3. For each $m \in \mathbb{N}, 0<s<t \leq 1, q \in[1, \infty[$,

$$
\left\|W_{s, t}^{(2)}-W(m)_{s, t}^{(2)}\right\|_{q} \leq C 2^{-m \mu}|t-s|^{2 H-\varepsilon},
$$

for some positive constants $C$ and any $\varepsilon \in] 0,2 H-\frac{1}{2}[$ and $\mu \in] 0, \frac{\varepsilon}{2}[$. 
Before proving this proposition, we give an equivalent expression for $W(m)_{s, t}^{2}$, as follows. The integration by parts formula of Malliavin calculus (see e.g. [8], Equation (1.49)) and (1.6) yield $W(m)_{s, t}^{(2)}=A_{s, t}^{1}(m)+A_{s, t}^{2}(m)$, with

$$
\begin{aligned}
& A_{s, t}^{1}(m)=\sum_{k=1}^{2^{m}} \int_{0}^{1} d u \mathbf{1}_{\Delta_{k}^{m}}(u) 2^{m} K^{*}\left(\mathbf{1}_{s, t]}(\cdot) \int_{\Delta_{k}^{m}} d B_{r}\left(W(m) .-W(m)_{s}\right)\right)(u), \\
& A_{s, t}^{2}(m)=\sum_{k=1}^{2^{m}} \int_{0}^{1} d u \mathbf{1}_{\Delta_{k}^{m}}(u) 2^{m} K^{*}\left(\mathbf{1}_{s, t]}(\cdot) \int_{\Delta_{k}^{m}} d r\left(K_{m}(\cdot, r)-K_{m}(s, r)\right)\right)(u) .
\end{aligned}
$$

By definition, for $r \in \Delta_{k}^{m}, K_{m}(t, r)=2^{m} \int_{\left.\Delta_{k}^{m} \cap 0, t\right]} K(t, u) d u=2^{m} K\left(\mathbf{1}_{\Delta_{k}^{m}}\right)(t)$. Since $h:=K\left(\mathbf{1}_{\Delta_{k}^{m}}\right) \in \mathcal{H}_{K}$, the duality relation given in [7], equation (58) and Lemma 3.3 yield

$$
\begin{aligned}
A_{s, t}^{2}(m) & =\sum_{k=1}^{2^{m}} \int_{0}^{1} d r \mathbf{1}_{\Delta_{k}^{m}}(r) 2^{2 m} \int_{0}^{1} d u \mathbf{1}_{\Delta_{k}^{m}}(u) K^{*}\left(\mathbf{1}_{] s, t]}(\cdot) K\left(\mathbf{1}_{\Delta_{k}^{m}}\right)_{s,}\right)(u) \\
& =\sum_{k=1}^{2^{m}} \int_{0}^{1} d r \mathbf{1}_{\Delta_{k}^{m}}(r) 2^{2 m} \int_{s}^{t} K\left(\mathbf{1}_{\Delta_{k}^{m}}\right)(d u)\left(K\left(\mathbf{1}_{\Delta_{k}^{m}}\right)(u)-K\left(\mathbf{1}_{\Delta_{k}^{m}}\right)(s)\right) \\
& =\sum_{k=1}^{2^{m}} \int_{0}^{1} d r \mathbf{1}_{\Delta_{k}^{m}}(r) 2^{2 m} \frac{\left(K\left(\mathbf{1}_{\Delta_{k}^{m}}\right)(t)-K\left(\mathbf{1}_{\Delta_{k}^{m}}\right)(s)\right)^{2}}{2} \\
& =\frac{1}{2} \int_{0}^{1} d r\left|K_{m}(t, r)-K_{m}(s, r)\right|^{2}=\frac{1}{2}\left\|W(m)_{s, t}^{(1)}\right\|_{2}^{2} .
\end{aligned}
$$

Thus, since $E\left|W_{t}-W_{s}\right|^{2}=|t-s|^{2 H}$, Schwarz's inequality, (3.14), (3.15) imply

$$
\left|A_{s, t}^{2}(m)-\frac{1}{2}\right| t-\left.s\right|^{2 H}\left|\leq C 2^{-m \varepsilon}\right| t-\left.s\right|^{2 H-\varepsilon},
$$

for some positive constant $C$ and $\varepsilon \in] 0, H[$.

Hence, in order to establish (2.6) it suffices to prove that for any small parameter $\varepsilon \in] 0,4 H-1[$ and $\mu \in] 0, \varepsilon[$,

$$
E\left(\left|\int_{0}^{1} K^{*}\left(\mathbf{1}_{] s, t]}(\cdot)\left(W .-W_{s}\right)\right)(u) d B_{u}-A_{s, t}^{1}(m)\right|^{2}\right) \leq C 2^{-m \mu}|t-s|^{4 H-\epsilon} .
$$

for all $m \geq 1$. We devote the next lemmas to the proof of this convergence, using the expression of the operator $K^{*}$ given in (2.3).

Lemma 2.4. For any $0 \leq s<t \leq 1, m \geq 1$, we set

$$
T_{1}(s, t)=\int_{0}^{s} d B_{u}\left(\int_{s}^{t}\left(W_{r}-W_{s}\right) K(d r, u)\right),
$$


$T_{1}(s, t, m)=\sum_{k=1}^{2^{m}} \int_{\Delta_{k}^{m}} d B_{r} 2^{m}\left(\int_{\left.\left.\Delta_{k}^{m} \cap\right] 0, s\right]} d u\left(\int_{s}^{t}\left(W(m)_{v}-W(m)_{s}\right) K(d v, u)\right)\right)$.

Then for any $\epsilon \in] 0,2 H[$ and $\mu \in] 0, \epsilon[$, there exists $C>0$ such that

$$
E\left(\left|T_{1}(s, t, m)-T_{1}(s, t)\right|^{2}\right) \leq C 2^{-m \mu}|t-s|^{4 H-\epsilon} .
$$

Proof. Assume $s \in \Delta_{I}^{m}, I \geq 1$; we consider the decomposition

$$
E\left(\left|T_{1}(s, t, m)-T_{1}(s, t)\right|^{2}\right) \leq C \sum_{j=1}^{3} \tau_{1, j}(s, t, m),
$$

with

$$
\begin{aligned}
\tau_{1,1}(s, t, m)= & \sum_{k \in\{1, I-1, I\}} E\left(\mid \int_{\Delta_{k}^{m}} d B_{r} 2^{m}\right. \\
& \left.\times\left.\left(\int_{\left.\Delta_{k}^{m} \cap j, s\right]} d u\left(\int_{s}^{t}\left(W(m)_{v}-W(m)_{s}\right) K(d v, u)\right)\right)\right|^{2}\right) \\
\tau_{1,2}(s, t, m)= & \sum_{k \in\{1, I-1, I\}} E\left(\left|\int_{\left.\left.\Delta_{k}^{m} \cap\right] 0, s\right]} d B_{r}\left(\int_{s}^{t}\left(W_{v}-W_{s}\right) K(d v, r)\right)\right|^{2}\right) \\
\tau_{1,3}(s, t, m)= & E\left(\mid \sum_{k=2}^{I-2} \int_{\Delta_{k}^{m}} d B_{r} 2^{m} \int_{\Delta_{k}^{m}} d u\left(\int_{s}^{t}\left(W(m)_{v}-W(m)_{s}\right) K(d v, u)\right.\right. \\
- & \left.\left.\int_{s}^{t}\left(W_{v}-W_{s}\right) K(d v, r)\right)\left.\right|^{2}\right)
\end{aligned}
$$

By Lemma 3.4, (3.4), Schwarz's inequality and (3.14), any term in the right hand-side of (2.12) is bounded as follows. Let $\varepsilon \in] 0,2 H[, \lambda \in] \frac{1-(2 H-\varepsilon)}{2}, \frac{1}{2}[$; then $2 H-3+2 \lambda<-1,1-2 \lambda-(2 H-\varepsilon)<0$ and

$$
\begin{aligned}
& E\left(\left|\int_{\Delta_{k}^{m}} d B_{r} 2^{m}\left(\int_{\left.\left.\Delta_{k}^{m} \cap\right] 0, s\right]} d u\left(\int_{s}^{t}\left(W(m)_{v}-W(m)_{s}\right) K(d v, u)\right)\right)\right|^{2}\right) \\
& \leq C \int_{\Delta_{k}^{m}} d r \int_{0}^{1} d \rho\left|2^{m} \int_{\left.\left.\Delta_{k}^{m} \cap\right] 0, s\right]} d u \int_{s}^{t}\left(K_{m}(v, \rho)-K_{m}(s, \rho)\right) K(d v, u)\right|^{2} \\
& \leq C \int_{\Delta_{k}^{m}} d r \int_{0}^{1} d \rho 2^{m} \int_{\left.\left.\Delta_{k}^{m} \cap\right] 0, s\right]} d u\left(\int_{s}^{t} d v|v-u|^{2 H-3+2 \lambda}\right) \\
& \quad \times\left(\int_{s}^{t} d v\left|K_{m}(v, \rho)-K_{m}(s, \rho)\right|^{2}|v-u|^{-2 \lambda}\right)
\end{aligned}
$$




$$
\begin{aligned}
& \leq C \int_{\left.\left.\Delta_{k}^{m} \cap\right] 0, s\right]} d u(s-u)^{2 H-2+2 \lambda}|t-s|^{2 H}\left(|t-u|^{1-2 \lambda}-|s-u|^{1-2 \lambda}\right) \\
& \leq C \int_{\left.\left.\Delta_{k}^{m} \cap\right] 0, s\right]} d u(s-u)^{2 H-2+2 \lambda}|t-s|^{2 H}|t-s|^{2 H-\varepsilon}|s-u|^{1-2 \lambda-(2 H-\varepsilon)} \\
& \leq C|t-s|^{4 H-\varepsilon} \int_{\left.\left.\Delta_{k}^{m} \cap\right] 0, s\right]} d u|s-u|^{\varepsilon-1} \leq C 2^{-m \varepsilon}|t-s|^{4 H-\varepsilon} .
\end{aligned}
$$

Each term of the right hand-side of (2.13) can be studied using a similar strategy. Thus we obtain for $\varepsilon \in] 0,2 H[$ :

$$
\tau_{1,1}(s, t, m)+\tau_{1,2}(s, t, m) \leq C 2^{-m \varepsilon}|t-s|^{4 H-\varepsilon} .
$$

Set for $s \geq 3 \cdot 2^{-m}$, and hence $I \geq 4$,

$$
\begin{aligned}
X_{r} & =\sum_{k=2}^{I-2} \mathbf{1}_{\Delta_{k}^{m}}(r) 2^{m} \int_{\Delta_{k}^{m}} d u\left(\int_{s}^{t}\left(W(m)_{v}-W(m)_{s}\right) K(d v, u)\right. \\
& \left.-\int_{s}^{t}\left(W_{v}-W_{s}\right) K(d v, r)\right) .
\end{aligned}
$$

Notice that $X_{r}=\int_{0}^{1} g(r, \rho) d B_{\rho}$, with

$$
\begin{aligned}
g(r, \rho) & =\sum_{k=2}^{I-2} \mathbf{1}_{\Delta_{k}^{m}}(r) 2^{m} \int_{\Delta_{k}^{m}} d u\left(\int_{s}^{t} K(d v, u)\left(K_{m}(v, \rho)-K_{m}(s, \rho)\right)\right. \\
& \left.-\int_{s}^{t} K(d v, r)(K(v, \rho)-K(s, \rho))\right) .
\end{aligned}
$$

Hence, by Lemma 3.4 and Schwarz's inequality, $\tau_{1,3}(s, t, m) \leq C\left(\tau_{1,3,1}(s, t, m)+\right.$ $\left.\tau_{1,3,2}(s, t, m)\right)$, with

$$
\begin{aligned}
\tau_{1,3,1}(s, t, m)= & \sum_{k=2}^{I-2} 2^{m} \int_{\Delta_{k}^{m}} d r \int_{\Delta_{k}^{m}} d u \int_{0}^{1} d \rho \\
\times & \left|\int_{s}^{t}\left(K_{m}(v, \rho)-K_{m}(s, \rho)\right)(K(d v, u)-K(d v, r))\right|^{2}, \\
\tau_{1,3,2}(s, t, m)= & \sum_{k=2}^{I-2} \int_{\Delta_{k}^{m}} d r \int_{0}^{1} d \rho \mid \int_{s}^{t} K(d v, r) \\
& \times\left.\left(K_{m}(v, \rho)-K_{m}(s, \rho)-K(v, \rho)+K(s, \rho)\right)\right|^{2} .
\end{aligned}
$$

Owing to (3.4), (3.7), we have for $\lambda \in] 0,1\left[, u, r \in \Delta_{k}^{m}\right.$,

$$
\begin{aligned}
& \left|\frac{\partial K}{\partial v}(v, u)-\frac{\partial K}{\partial v}(v, r)\right| \\
& \quad \leq C\left|\frac{\partial K}{\partial v}(v, u)-\frac{\partial K}{\partial v}(v, r)\right|^{\lambda}\left(\left|\frac{\partial K}{\partial v}(v, u)\right|^{1-\lambda}+\left|\frac{\partial K}{\partial v}(v, r)\right|^{1-\lambda}\right)
\end{aligned}
$$




$$
\leq C 2^{-m \lambda}|v-(u \vee r)|^{H-\frac{3}{2}}\left[(u \wedge r)^{-1}+|v-(u \vee r)|^{-1}\right]^{\lambda} .
$$

Thus, taking $\lambda:=H$ yields $\tau_{1,3,1}(s, t, m) \leq C 2^{-2 m H} \sum_{j=1}^{2} \tau_{1,3,1, j}(s, t, m)$, with

$$
\begin{aligned}
\tau_{1,3,1,1}(s, t, m)= & \sum_{k=2}^{I-2} 2^{m} \int_{\Delta_{k}^{m}} d r \int_{\Delta_{k}^{m}} d u \int_{0}^{1} d \rho\left(\int_{s}^{t} d v\left|K_{m}(v, \rho)-K_{m}(s, \rho)\right|\right. \\
& \left.\times|v-(u \vee r)|^{H-\frac{3}{2}}(u \wedge r)^{-H}\right)^{2}, \\
\tau_{1,3,1,2}(s, t, m)= & \sum_{k=2}^{I-2} 2^{m} \int_{\Delta_{k}^{m}} d r \int_{\Delta_{k}^{m}} d u \int_{0}^{1} d \rho\left(\int_{s}^{t} d v\left|K_{m}(v, \rho)-K_{m}(s, \rho)\right|\right. \\
& \left.\times|v-(u \vee r)|^{-\frac{3}{2}}\right)^{2} .
\end{aligned}
$$

Let $a=2-\epsilon$, with $\epsilon \in] 0,2 H$. Schwarz's inequality along with (3.14) yield

$$
\begin{aligned}
\tau_{1,3,1,1}(s, t, m) \leq & C \sum_{k=2}^{I-2} 2^{m} \int_{\Delta_{k}^{m}} d r \int_{\Delta_{k}^{m}} d u\left(\int_{s}^{t} d v|v-(u \vee r)|^{-a} d v\right) \\
& \times\left(\int_{s}^{t} d v|v-s|^{4 H-3+a}|u \wedge r|^{-2 H}\right) \\
\leq & C|t-s|^{4 H-\epsilon} \int_{t_{1}^{m}}^{t_{I-2}^{m}} d u\left(s-\bar{u}_{m}\right)^{\epsilon-1}\left(\underline{u}_{m}\right)^{-2 H} \\
\leq & C|t-s|^{4 H-\epsilon} s^{\epsilon-2 H} \leq|t-s|^{4 H-\epsilon} 2^{-m(\epsilon-2 H)} .
\end{aligned}
$$

Indeed, $\int_{s}^{t} d v|v-(u \vee r)|^{-2+\epsilon} \leq C\left(s-\bar{u}_{m}\right)^{\epsilon-1}$ for $\bar{u}_{m}$ defined by (3.13). Let $\epsilon \in] 0,2 H$ [ using Schwarz's inequality and (3.14), we obtain

$$
\begin{aligned}
\tau_{1,3,1,2}(s, t, m) \leq & C \sum_{k=2}^{I-2} 2^{m} \int_{\Delta_{k}^{m}} d r \int_{\Delta_{k}^{m}} d u\left(\int_{s}^{t} d v|v-(u \vee r)|^{-2-2 H+\varepsilon}\right) \\
& \times\left(\int_{s}^{t} d v|v-(u \vee r)|^{2 H-\varepsilon-1}|v-s|^{2 H}\right) \\
\leq & C|t-s|^{4 H-\varepsilon} \int_{t_{1}^{m}}^{t_{I-2}^{m}} d u \int_{s}^{t} d v\left(v-\bar{u}_{m}\right)^{-2-2 H+\varepsilon} \\
\leq & C|t-s|^{4 H-\varepsilon} \int_{t_{1}^{m}}^{t_{I-2}^{m}} d u\left(s-\bar{u}_{m}\right)^{-1-2 H+\varepsilon} \\
\leq & C|t-s|^{4 H-\varepsilon} 2^{-m(\varepsilon-2 H)}
\end{aligned}
$$

From (2.17), (2.18) we deduce that for $\epsilon \in] 0,2 H[$,

$$
\tau_{1,3,1}(s, t, m) \leq C|t-s|^{4 H-\epsilon} 2^{-m \epsilon} .
$$

Let $\delta \in] 0,2 H[, \alpha \in] 0,2 H[, \lambda \in] 0,1[$ and $\mu \in] \frac{1}{2}, 1-H[$. Notice that for these choices, $-2 \mu+1-2 H+\delta<0$. Hölder's inequality together with (3.14) and 

(3.15) yield for any $\lambda \in] 0,1[$,

$$
\tau_{1,3,2}(s, t, m) \leq C \tau_{1,3,2,1}(s, t, m)^{\lambda} \tau_{1,3,2,2}(s, t, m)^{1-\lambda},
$$

where

$$
\begin{aligned}
& \tau_{1,3,2,1}(s, t, m)=\int_{t_{1}^{m}}^{t_{I-2}^{m}} d r\left(\int_{s}^{t} d v(v-r)^{2 H-3+2 \mu}\right)\left(\int_{s}^{t} d v(v-r)^{-2 \mu}(v-s)^{2 H}\right), \\
& \tau_{1,3,2,2}(s, t, m)=\int_{t_{1}^{m}}^{t_{I-2}^{m}} d r\left(\int_{s}^{t} d v(v-r)^{2 H-3+2 \mu}\right)\left(\int_{s}^{t} d v(v-r)^{-2 \mu} 2^{-2 m H}\right) .
\end{aligned}
$$

For the first term we have

$$
\begin{aligned}
\tau_{1,3,2,1}(s, t, m) & \leq C|t-s|^{4 H-\delta} \int_{t_{1}^{m}}^{t_{I-2}^{m}} d r(s-r)^{2 H-2+2 \mu}(s-r)^{-2 \mu+1-2 H+\delta} \\
& \leq C|t-s|^{4 H-\delta}
\end{aligned}
$$

while for the second one, we obtain

$\tau_{1,3,2,2}(s, t, m) \leq C 2^{-2 m H}|t-s|^{2 H-\alpha} \int_{t_{1}^{m}}^{t_{I-2}^{m}} d r(s-r)^{2 H-2+2 \mu}(s-r)^{-2 \mu+1-2 H+\alpha}$.

Consequently,

$$
\tau_{1,3,2}(s, t, m) \leq C|t-s|^{(4 H-\delta) \lambda+(2 H-\alpha)(1-\lambda)} 2^{-2 m H(1-\lambda)} .
$$

Take $\alpha, \delta$ arbitrarily small and $1-\lambda=\frac{\epsilon-H \delta}{2 H+\alpha}$. Then for $\beta<\epsilon<2 H$, we have proved that

$$
\tau_{1,3,2}(s, t, m) \leq C|t-s|^{4 H-\epsilon} 2^{-m \beta} .
$$

This inequality, together with (2.15) and (2.19) yields (2.11).

Lemma 2.5. For any $0 \leq s<t \leq 1$, set

$$
\begin{aligned}
T_{2}(s, t) & =\int_{s}^{t} d B_{u} K(t, u)\left(W_{u}-W_{s}\right), \\
T_{2}(s, t, m) & =\sum_{k=1}^{2^{m}} \int_{\Delta_{k}^{m}} d B_{r} 2^{m}\left(\int_{\left.\left.\Delta_{k}^{m} \cap\right] s, t\right]} d u K(t, u)\left(W(m)_{u}-W(m)_{s}\right)\right) .
\end{aligned}
$$

Then, for $b \in] 0,2 H[$, there exists a constant $C>0$ such that for each $m \geq 1$

$$
E\left(\left|T_{2}(s, t, m)-T_{2}(s, t)\right|^{2}\right) \leq C 2^{-m b}|t-s|^{4 H-b} .
$$

Proof. Let $s \in \Delta_{I}^{m}, t \in \Delta_{J}^{m}$. We have

$$
E\left(\left|T_{2}(s, t, m)-T_{2}(s, t)\right|^{2}\right) \leq C \sum_{j=1}^{3} T_{2, j}(s, t, m),
$$

with for $\mathcal{I}=\{I, I+1, J-2, J-1 J\}$

$$
T_{2,1}(s, t, m)=\sum_{k \in \mathcal{I}} E\left(\left|\int_{\Delta_{k}^{m}} d B_{r} 2^{m} \int_{\left.\left.\Delta_{k}^{m} \cap\right] s, t\right]} d u K(t, u)\left(W(m)_{u}-W(m)_{s}\right)\right|^{2}\right) \text {, }
$$




$$
\begin{aligned}
T_{2,2}(s, t, m)= & \sum_{k \in \mathcal{I}} E\left(\left|\int_{\left.\left.\Delta_{k}^{m} \cap\right] s, t\right]} d B_{r} K(t, r)\left(W_{r}-W_{s}\right)\right|^{2}\right), \\
T_{2,3}(s, t, m)=E(\mid & \sum_{k=I+2}^{J-3} \int_{\Delta_{k}^{m}} d B_{r}\left[2^{m} \int_{\left.\left.\Delta_{k}^{m} \cap\right] s, t\right]} d u K(t, u)\left(W(m)_{u}-W(m)_{s}\right)\right. \\
& \left.\left.-K(t, r)\left(W_{r}-W_{s}\right)\right]\left.\right|^{2}\right) .
\end{aligned}
$$

Owing to Lemma 3.4 applied to the Gaussian process

$$
X_{r}:=\mathbf{1}_{\Delta_{k}^{m}}(r) \int_{0}^{1} d B_{\rho}\left(2^{m} \int_{\left.\left.\Delta_{k}^{m} \cap\right] s, t\right]} d u K(t, u)\left(K_{m}(u, \rho)-K_{m}(s, \rho)\right)\right)
$$

and Schwarz's inequality, we have for any $k=1, \cdots, 2^{m}$,

$$
\begin{aligned}
T(s, t, m, k):= & E\left(\left|\int_{\Delta_{k}^{m}} d B_{r} 2^{m} \int_{\left.\left.\Delta_{k}^{m} \cap\right] s, t\right]} d u K(t, u)\left(W(m)_{u}-W(m)_{s}\right)\right|^{2}\right) \\
\leq & C 2^{2 m} \int_{\Delta_{k}^{m}} d r \int_{0}^{1} d \rho\left(\int_{\left.\left.\Delta_{k}^{m} \cap\right] s, t\right]} d u K^{2}(t, u)\right) \\
& \times\left(\int_{\left.\left.\Delta_{k}^{m} \cap\right] s, t\right]} d u\left|K_{m}(u, \rho)-K_{m}(s, \rho)\right|^{2}\right) .
\end{aligned}
$$

Let $k=I, I+1$; since $\int_{\left.\left.\Delta_{k}^{m} \cap\right] s, t\right]} d u K^{2}(t, u) \leq \int_{] s, t]} d u K^{2}(t, u) \leq C|t-s|^{2 H}$, we have for any $b \in] 0,2 H[$,

$$
\begin{aligned}
T(s, t, m, k) & \leq C 2^{m}|t-s|^{2 H}\left(\int_{\left.\left.\Delta_{k}^{m} \cap\right] s, t\right]} d u|u-s|^{2 H}\right) \\
& \leq C 2^{m}|t-s|^{4 H-b} \int_{\left.\left.\Delta_{k}^{m} \cap\right] s, t\right]} d u|u-s|^{b} \leq C 2^{-m b}|t-s|^{4 H-b} .
\end{aligned}
$$

Let $k=J-2, J-1, J$ with $J-2>I+1$ then for $u \in \Delta_{k}^{m}$, 3.5 implies $|K(t, u)|^{2} \leq C|t-u|^{2 H-1}$ and $|t-u| \leq C 2^{-m}$; we obtain for $\left.b \in\right] 0,2 H[$,

$$
\begin{aligned}
T(s, t, m, k) & \leq C 2^{m}\left(\int_{\left.\left.\Delta_{k}^{m} \cap\right] s, t\right]} d u|t-u|^{2 H-1-b} 2^{-m b} d u\right)\left(\int_{\left.\left.\Delta_{k}^{m} \cap\right] s, t\right]} d u|u-s|^{2 H}\right) \\
& \leq C|t-s|^{4 H-b} 2^{-m b} .
\end{aligned}
$$

We therefore have proved that for $b \in] 0,2 H[$,

$$
T_{2,1}(s, t, m) \leq C 2^{-b m}|t-s|^{4 H-b}
$$


The analysis of the term $T_{2,2}(s, t, m)$ is easier. Indeed, the isometry property of the stochastic integral yields for any $k=1, \cdots, 2^{m}$,

$$
E\left(\left|\int_{\left.\left.\Delta_{k}^{m} \cap\right] s, t\right]} d B_{r} K(t, r)\left(W_{r}-W_{s}\right)\right|^{2}\right)=C \int_{\left.\left.\Delta_{k}^{m} \cap\right] s, t\right]} d r K^{2}(t, r)|r-s|^{2 H} .
$$

For the particular values of $k \in \mathcal{I}$, the right hand-side of (2.22) can be analyzed following similar ideas as for $T_{2,1}(s, t, m)$, which yields for $b \in$ ] $0,2 H[$

$$
T_{2,2}(s, t, m) \leq C 2^{-m b}|t-s|^{4 H-b} .
$$

We now study $T_{2,3}(s, t, m)$ and note that $T_{2,3}(s, t, m)=0$ if $|t-s| \leq 2^{-m}$. Thus, we may assume that $t-s \geq 2^{-m}$. First, we apply Lemma 3.4 and obtain

$$
T_{2,3}(s, t, m) \leq C\left(T_{2,3,1}(s, t, m)+T_{2,3,2}(s, t, m)\right)
$$

where

$$
\begin{aligned}
T_{2,3,1}(s, t, m)= & \int_{\bar{s}_{m}}^{\underline{t}_{m}-2^{1-m}} d r \int_{0}^{1} d \rho \mid 2^{m} \int_{\underline{r}_{m}}^{\bar{r}_{m}} d u(K(t, u)-K(t, r)) \\
& \times\left.\left(K_{m}(u, \rho)-K_{m}(s, \rho)\right)\right|^{2} \\
T_{2,3,2}(s, t, m)= & \int_{\bar{s}_{m}}^{\underline{t}_{m}-2^{1-m}} d r \int_{0}^{1} d \rho \mid 2^{m} \int_{\underline{\underline{r}}_{m}}^{\bar{r}_{m}} d u K(t, r) \\
& \times\left.\left(\left[K_{m}(u, \rho)-K_{m}(s, \rho)\right]-[K(r, \rho)-K(s, \rho)]\right)\right|^{2} .
\end{aligned}
$$

By Schwarz's inequality and (3.14), for $b \in] 0,2 H[$,

$$
\begin{aligned}
T_{2,3,1}(s, t, m) & \leq \int_{\bar{s}_{m}}^{\underline{t}_{m}-2^{1-m}} d r 2^{m} \int_{\underline{r}_{m}}^{\bar{r}_{m}} d u|K(t, u)-K(t, r)|^{2}|u-s|^{2 H} \\
& \leq C|t-s|^{2 H} \int_{\bar{s}_{m}}^{\underline{t}_{m}-2^{1-m}} d r 2^{m} \int_{\underline{r}_{m}}^{\bar{r}_{m}} d u|K(t, u)-K(t, r)|^{2} \\
& \leq C 2^{-2 m H}|t-s|^{2 H} \leq C 2^{-m b}|t-s|^{4 H-b}
\end{aligned}
$$

where the last inequalities follow from (3.19) and $|t-s| \geq 2^{-m}$.

Owing to (3.15), we have for $u \in\left[\underline{r}_{m}, \bar{r}_{m}\right]$

$$
\begin{aligned}
\int_{0}^{1} d \rho\left|K_{m}(s, \rho)-K(s, \rho)\right|^{2} & \leq C 2^{-2 m H} \\
\int_{0}^{1} d \rho\left|K_{m}(u, \rho)-K(r, \rho)\right|^{2} \leq & C \int_{0}^{1} d \rho\left(\left|K_{m}(u, \rho)-K(u, \rho)\right|^{2}\right. \\
& \left.+|K(u, \rho)-K(r, \rho)|^{2}\right) \leq C 2^{-2 m H}
\end{aligned}
$$


Schwarz's inequality, along with (3.5) and the above estimates yield

$$
\begin{aligned}
T_{2,3,2}(s, t, m) & \leq C \int_{\bar{s}_{m}}^{\underline{t}_{m}-2^{1-m}} d r 2^{-2 m H}\left(|r|^{2 H-1}+|t-r|^{2 H-1}\right) \\
& \leq C 2^{-2 m H}\left(t^{2 H}-s^{2 H}+|t-s|^{2 H}+2^{-2 m H}\right) \\
& \leq C 2^{-2 m H}|t-s|^{2 H} \leq C 2^{-m b}|t-s|^{4 H-b}
\end{aligned}
$$

for $b \in] 0,2 H[$. Indeed, for each $H \in] 0, \frac{1}{2}\left[\right.$, and $s<t, t^{2 H}-s^{2 H} \leq(t-s)^{2 H}$ and we are assuming that $2^{-m}<|t-s|$. Thus, (2.20) is proved.

Lemma 2.6. For any $0 \leq s<t \leq 1$, set

$$
\begin{aligned}
T_{3}(s, t) & =\int_{s}^{t} d B_{u} \int_{u}^{t} K(d r, u)\left(W_{r}-W_{u}\right) \\
T_{3}(s, t, m) & =\sum_{k=1}^{2^{m}} 2^{m} \int_{\Delta_{k}^{m}} d B_{r} \int_{\left.\left.\Delta_{k}^{m} \cap\right] s, t\right]} d u \int_{u}^{t} K(d v, u)\left(W(m)_{v}-W(m)_{u}\right) .
\end{aligned}
$$

There exists a positive constant $C$ such that, for any $\epsilon \in] 0,4 H-1[$

$$
E\left(\left|T_{3}(s, t, m)-T_{3}(s, t)\right|^{2}\right) \leq C 2^{-m \epsilon}|t-s|^{4 H-\epsilon}
$$

for each $m \geq 1$.

Proof. Assume $s \in \Delta_{I}^{m}, t \in \Delta_{J}^{m}$; we consider the upper bound

$$
E\left(\left|T_{3}(s, t, m)-T_{3}(s, t)\right|^{2}\right) \leq C \sum_{j=1}^{3} T_{3, j}(s, t, m),
$$

where for $\mathcal{J}=\{I, I+1, J-1, J\}$

$$
\begin{aligned}
T_{3,1}(s, t, m)= & \sum_{k \in \mathcal{J}} E\left(\mid 2^{m} \int_{\Delta_{k}^{m}} d B_{r} \int_{\left.\left.\Delta_{k}^{m} \cap\right] s, t\right]} d u \int_{u}^{t} K(d v, u)\right. \\
& \left.\times\left.\left(W(m)_{v}-W(m)_{u}\right)\right|^{2}\right) \\
T_{3,2}(s, t, m)= & \sum_{k \in \mathcal{J}} E\left(\left|\int_{\left.\left.\Delta_{k}^{m} \cap\right] s, t\right]} d B_{r} \int_{r}^{t} K(d v, r)\left(W_{v}-W_{r}\right)\right|^{2}\right) \\
T_{3,3}(s, t, m)= & E\left(\mid \sum_{k=I+2}^{J-2} 2^{m} \int_{\Delta_{k}^{m}} d B_{r} \int_{\Delta_{k}^{m}} d u\right. \\
& \left.\times\left.\left(\int_{u}^{t} K(d v, u)\left(W(m)_{v}-W(m)_{u}\right)-\int_{r}^{t} K(d v, r)\left(W_{v}-W_{r}\right)\right)\right|^{2}\right) .
\end{aligned}
$$

Lemma 3.4 along with Schwarz's inequality yield for each term of the sum in the right hand side of (2.25) the upper bound

$$
C \int_{\Delta_{k}^{m}} d r \int_{0}^{1} d \rho 2^{m} \int_{\left.\left.\Delta_{k}^{m} \cap\right] s, t\right]} d u\left(\int_{u}^{t} K(d v, u)\left(K_{m}(v, \rho)-K_{m}(u, \rho)\right)\right)^{2} .
$$


Fix $a \in] 2-4 H, 1]$. From Schwarz's inequality, (3.4) and (3.14) we deduce the following estimates for this integral:

$$
\begin{aligned}
& C \int_{\Delta_{k}^{m}} d r 2^{m} \int_{\left.\left.\Delta_{k}^{m} \cap\right] s, t\right]} d u\left(\int_{u}^{t} d v|v-u|^{-a}\right)\left(\int_{u}^{t}|v-u|^{4 H-3+a}\right) \\
& \quad \leq C\left(2^{-m} \wedge|t-s|\right)|t-s|^{4 H-1} .
\end{aligned}
$$

A similar analysis yields the same result for each term in the right hand-side of (2.26). Consequently,

$$
T_{3,1}(s, t, m)+T_{3,2}(s, t, m) \leq C\left(2^{-m} \wedge|t-s|\right)|t-s|^{4 H-1} .
$$

If $|t-s| \leq 2^{-m}$ then $T_{3,3}(s, t, m)=0$. Hence, let us assume that $t-s \geq 2^{-m}$; in this case $T_{3,3}(s, t, m)$ is equal to $E\left(\int_{0}^{1} d B_{r} X_{r}\right)^{2}$, with $X_{r}=\int_{0}^{1} d B_{\rho} g(r, \rho)$, and

$$
\begin{aligned}
g(r, \rho)= & \sum_{k=I+2}^{J-2} \mathbf{1}_{\Delta_{k}^{m}}(r) 2^{m} \int_{\Delta_{k}^{m}} d u\left[\int_{u}^{t} K(d v, u)\left(K_{m}(v, \rho)-K_{m}(u, \rho)\right)\right. \\
& \left.-\int_{r}^{t} K(d v, r)(K(v, \rho)-K(r, \rho))\right] .
\end{aligned}
$$

We at first study the contribution to $T_{3,3}(s, t, m)$ of the integrands

$$
\begin{aligned}
& g_{1}(r, \rho)=\sum_{k=I+2}^{J-2} \mathbf{1}_{\Delta_{k}^{m}}(r) 2^{m} \int_{\Delta_{k}^{m}} d u \int_{u}^{u \vee r} K(d v, u)\left(K_{m}(v, \rho)-K_{m}(u, \rho)\right), \\
& g_{2}(r, \rho)=\sum_{k=I+2}^{J-2} \mathbf{1}_{\Delta_{k}^{m}}(r) 2^{m} \int_{\Delta_{k}^{m}} d u \int_{r}^{u \vee r} K(d v, r)(K(v, \rho)-K(r, \rho)),
\end{aligned}
$$

which we denote by $T_{3,3, j}(s, t, m), j=1,2$. Actually, both are similar and therefore we only study the first one. Lemma 3.4. (3.4), (3.14) and Schwarz's inequality imply, for each $a \in] 2-4 H, 1]$,

$$
\begin{aligned}
T_{3,3,1}(s, t, m) & \leq C \sum_{k=I+2}^{J-2} 2^{m} \int_{\Delta_{k}^{m}} d r \int_{\Delta_{k}^{m}} d u \int_{u}^{u \vee r} d v|v-u|^{-a} \int_{u}^{u \vee r} d v|v-u|^{4 H-3+a} \\
& \leq C 2^{-m(4 H-1)}|t-s|
\end{aligned}
$$

We end the analysis of the term $T_{3,3}(s, t, m)$ by studying the contribution of $T_{3,3,3}((s, t, m)$ defined in terms of the integrand

$$
\begin{aligned}
g_{3}(r, \rho)= & \sum_{k=I+2}^{J-2} \int_{\Delta_{k}^{m}} d r 2^{m} \int_{\Delta_{k}^{m}} d u \int_{u \vee r}^{t}\left[K(d v, u)\left(K_{m}(v, \rho)-K_{m}(u, \rho)\right)\right. \\
& -K(d v, r)(K(v, \rho)-K(r, \rho))] .
\end{aligned}
$$

Notice that $g_{3}(r, \rho)$ is the sum of two analogous terms where the set $\Delta_{k}^{m}$ of the integral with respect to the variable $u$ is replaced by $\underline{r}_{m}, r\left[,\left[r, \bar{r}_{m}[\right.\right.$, 
respectively. Again, the contribution of both terms is similar, so that we concentrate on the first one. That is, we consider

$$
\begin{aligned}
T_{3,3,3}^{+}(s, t, m):= & E\left(\mid \sum_{k=I+2}^{J-2} 2^{m} \int_{\Delta_{k}^{m}} d B_{r} \int_{\left[\underline{x}_{m}, r[\right.} d u \int_{r}^{t}[K(d v, u)\right. \\
& \left.\left.\times\left(W(m)_{v}-W(m)_{u}\right)-K(d v, r)\left(W_{v}-W_{r}\right)\right]\left.\right|^{2}\right) .
\end{aligned}
$$

As before, all the arguments rely on Lemma 3.4 (3.4), (3.14), a suitable factorization of the integrands along with Schwarz's inequality. In order to deal with the singularity at $v=r$, we first replace the integral with respect to the variable $v$ by $\int_{r}^{\bar{r}_{m}+2^{-m}}$. Given $\left.a \in\right] 2-4 H, 1[$, the corresponding contribution to $T_{3,3,3}^{+}(s, t, m)$ is bounded by

$$
\begin{aligned}
& C \sum_{k=I+2}^{J-2} 2^{m} \int_{\Delta_{k}^{m}} d r \int_{\left[\underline{r}_{m}, r[\right.} d u \int_{0}^{1} d \rho\left(\mid \int_{r}^{\bar{r}_{m}+2^{-m}} K(d v, u)\right. \\
& \left.\quad \times\left.\left(K_{m}(v, \rho)-K_{m}(u, \rho)\right)\right|^{2}+\left|\int_{r}^{\bar{r}_{m}+2^{-m}} K(d v, r)(K(v, \rho)-K(r, \rho))\right|^{2}\right) \\
& \leq C \sum_{k=I+2}^{J-2} 2^{m} \int_{\Delta_{k}^{m}} d r \int_{\underline{\underline{r}}_{m}, r[} d u \int_{r}^{\bar{r}_{m}+2^{-m}} d v|v-r|^{-a} \int_{r}^{\bar{r}_{m}+2^{-m}} d v|v-r|^{4 H-3+a} \\
& \leq C 2^{-m(4 H-1)}|t-s| .
\end{aligned}
$$

Let us finally consider the range $] r_{m}+2^{-m}, t[$ for the variable $v$. We have to study two terms:

$$
\begin{aligned}
M_{1}(s, t, m)= & \sum_{k=I+2}^{J-2} 2^{m} \int_{\Delta_{k}^{m}} d r \int_{\left[\underline{r}_{m}, r[\right.} d u \int_{0}^{1} d \rho\left(\int_{\bar{r}_{m}+2^{-m}}^{t} d v\right. \\
& \left.\times\left|K_{m}(v, \rho)-K_{m}(u, \rho)\right|\left|\frac{\partial K}{\partial v}(v, u)-\frac{\partial K}{\partial v}(v, r)\right|\right)^{2}, \\
M_{2}(s, t, m)= & \sum_{k=I+2}^{J-2} 2^{m} \int_{\Delta_{k}^{m}} d r \int_{\left[\underline{r}_{m}, r[\right.} d u \int_{0}^{1} d \rho\left(\int_{\bar{r}_{m}+2^{-m}}^{t} d v\left|\frac{\partial K}{\partial v}(v, r)\right|\right. \\
& \left.\times\left[\left(K_{m}(v, \rho)-K_{m}(u, \rho)\right)-(K(v, \rho)-K(r, \rho))\right]\right)^{2} .
\end{aligned}
$$

For $M_{1}(s, t, m)$, we proceed in a similar way as for the term $\tau_{1,3,1}(s, t, m)$ in Lemma 2.4 as follows. By means of (2.16) we obtain for $\lambda \in] 0,1[$ $M_{1}(s, t, m) \leq C 2^{-2 m \lambda}\left(M_{1,1}(s, t, m)+M_{1.2}(s, t, m)\right)$, with

$$
\begin{aligned}
M_{1,1}(s, t, m)= & \sum_{k=I+2}^{J-2} 2^{m} \int_{\Delta_{k}^{m}} d r \int_{\left[\underline{r}_{m}, r[\right.} d u u^{-2 \lambda} \int_{0}^{1} d \rho\left(\int_{\bar{r}_{m}+2^{-m}}^{t} d v\right. \\
& \left.\left|K_{m}(v, \rho)-K_{m}(u, \rho)\right||v-r|^{H-\frac{3}{2}}\right)^{2}
\end{aligned}
$$




$$
\begin{aligned}
M_{1,2}(s, t, m)= & \sum_{k=I+2}^{J-2} 2^{m} \int_{\Delta_{k}^{m}} d r \int_{\left[\underline{r}_{m}, r[\right.} d u \int_{0}^{1} d \rho\left(\int_{\bar{r}_{m}+2^{-m}}^{t} d v\right. \\
& \left.\left|K_{m}(v, \rho)-K_{m}(u, \rho)\right| v-\left.r\right|^{H-\frac{3}{2}-\lambda}\right)^{2} .
\end{aligned}
$$

Let $a \in] 2-4 H, 1[, \lambda \in] 0, \frac{1}{2}\left[\right.$. Since $t-s \geq 2^{-m}$, for $u \in\left[\underline{r}_{m}, r[\right.$, we have

$$
\int_{\bar{r}_{m}+2^{-m}}^{t} d v|v-r|^{2 H-3+a}|v-u|^{2 H} \leq C|t-r|^{4 H+a-2} .
$$

Consequently, since $r \geq u \geq \underline{r}_{m} \geq t_{I+1}$ implies $u \geq \frac{r}{2}$

$$
\begin{gathered}
M_{1,1}(s, t, m) \leq C \sum_{k=I+2}^{J-2} 2^{m} \int_{\Delta_{k}^{m}} d r \int_{\underline{\underline{r}}_{m}, r[} d u u^{-2 \lambda}\left(\int_{\bar{r}_{m}+2^{-m}}^{t} d v|v-r|^{-a}\right) \\
\times\left(\int_{\bar{r}_{m}+2^{-m}}^{t} d v|v-r|^{2 H-3+a}|v-u|^{2 H}\right) \\
\leq C \int_{s}^{t} r^{-2 \lambda}|t-s|^{4 H-1} d r \leq C|t-s|^{4 H-2 \lambda}
\end{gathered}
$$

Analogously, for $b \in] 2+2 \lambda-4 H, 1[, \lambda \in] 0,2 H-\frac{1}{2}\left[\right.$ and $|t-s| \geq 2^{-m}$

$$
\begin{aligned}
M_{1,2}(s, t, m) \leq & C \sum_{k=I+2}^{J-2} 2^{m} \int_{\Delta_{k}^{m}} d r \int_{\underline{\underline{r}}_{m}, r[} d u\left(\int_{\bar{r}_{m}+2^{-m}}^{t} d v|v-r|^{-b}\right) \\
& \times\left(\int_{\bar{r}_{m}+2^{-m}}^{t} d v|v-r|^{2 H-3-2 \lambda+b}|v-u|^{2 H}\right) \\
\leq & C \int_{s}^{t}|t-r|^{4 H-1-2 \lambda} d r=C|t-s|^{4 H-2 \lambda} .
\end{aligned}
$$

Finally, if we additionally use (3.15), we obtain for $a \in] 2-4 H, 1[$

$$
\begin{aligned}
M_{2}(s, t, m) \leq & C \sum_{k=I+2}^{J-2} 2^{m} \int_{\Delta_{k}^{m}} d r \int_{\underline{\underline{r}}_{m}, r[} d u\left(\int_{r}^{t} d v|v-r|^{-a}\right) \\
& \times\left(\int_{\underline{r}_{m}+2^{-m}}^{t} d v|v-r|^{2 H-3+a} 2^{-2 m H}\right) \\
\leq & C \int_{s}^{t}|t-r|^{1-a} 2^{-m(4 H-2+a)} d r \leq C 2^{-m b}|t-s|^{4 H-b}
\end{aligned}
$$

for $b \in] 0,4 H-1[$. We easily check that (2.24) follows from (2.27)-(2.32).

Proof of Proposition 2.3 We remark that Lemmas 2.4 to 2.6 yield the upper bound (2.10). Therefore, for $q=2$, (2.6) follows from (2.9) and (2.10). The hypercontractivity inequality yields the validity of the same inequality for any $q \in] 2, \infty[$.

Proof of Theorem [2.1] 
Let $H \in] \frac{1}{2}, 1[$ and $p \in] \frac{1}{H}, 2\left[\right.$. The convergence of $\tilde{d}_{p}(\mathbf{W}(\mathbf{m}), \mathbf{W})$ to zero in $L^{q}(\Omega)$ is a consequence of (2.2) and the usual version of the GarsiaRademich-Rumsey lemma (see e.g. 9], Theorem 2.1.3).

Consider the metric space $\left(\mathcal{G}_{p}, \tilde{d}_{p}\right)$. The canonical embedding $\mathcal{H}^{H} \hookrightarrow \mathcal{G}_{p}$ is continuous. Indeed, let $\dot{h}_{i}, i=1,2$, belong to $L^{2}([0,1])$. Then for $h_{i}()=$. $\int_{0} K(., r) \dot{h}_{i}(r) d r$ and $0 \leq s<t \leq 1$,

$$
\left|\left(h_{1}\right)_{s, t}^{(1)}-\left(h_{2}\right)_{s, t}^{(1)}\right| \leq|t-s|^{H}\left\|\dot{h}_{1}-\dot{h}_{2}\right\|_{2} \leq|t-s|^{\frac{1}{p}}\left\|h_{1}-h_{2}\right\|_{\mathcal{H}^{H}} .
$$

Consequently, the preceding convergence shows that $\left(\mathcal{G}_{p}, \mathcal{H}^{H}, P^{H}\right)$ is an abstract Wiener space.

Let now $H \in] \frac{1}{4}, \frac{1}{2}$ [. We follow the outline of the proof of Lemma 3 in [3], but refer to the extension of the Garsia-Rademich-Rumsey lemma stated in the Lemma 3.5 .

Fix $p \in] 2,4[$ such that $p H>1$. We shall prove that there exists $\theta>0$ such that for every $q \in[1, \infty[$,

$$
E\left(\left|\tilde{d}_{p}(\mathbf{W}, \mathbf{W}(\mathbf{m}))\right|^{q}\right) \leq C_{q} 2^{-m \theta q} .
$$

Indeed, for a fixed $q \in\left[1, \infty\left[\right.\right.$, let $M>q$ and $N=2 M$ satisfy $N>\frac{p}{2(H p-1)}$. Let $\alpha, \beta>0$ defined by $\alpha=\frac{2}{p}+\frac{1}{M}, \beta=\frac{1}{p}+\frac{1}{N}$.

By virtue of (2.1) and (2.6), we easily check that the random variables

$$
\begin{aligned}
& A_{1}(m):=\int_{0}^{1} \int_{0}^{1} d s d t 1_{\{s<t\}} \frac{\left|W_{s, t}^{(1)}-W(m)_{s, t}^{(1)}\right|^{2 N}}{|t-s|^{2 N \beta}}, \\
& A_{2}(m):=\int_{0}^{1} \int_{0}^{1} d s d t 1_{\{s<t\}} \frac{\left|W_{s, t}^{(2)}-W(m)_{s, t}^{(2)}\right|^{2 M}}{|t-s|^{2 M \alpha}},
\end{aligned}
$$

satisfy

$$
E\left(A_{1}(m)\right) \leq C 2^{-m \mu 2 N}, E\left(A_{2}(m)\right) \leq C 2^{-m \mu 2 M},
$$

for some $\mu>0$.

Furthermore, the hypercontractivity property and the inequality (3.14) imply that for $0 \leq s<t \leq 1$ and $q \in[1, \infty[$,

$$
\sup _{m}\left(\left\|W_{s, t}^{(1)}\right\|_{q}+\left\|W(m)_{s, t}^{(1)}\right\|_{q}\right) \leq C|t-s|^{H} .
$$

This yields

$$
\sup _{m} E(\eta(m)) \leq C
$$

where

$$
\eta(m):=\int_{0}^{1} \int_{0}^{1} d s d t 1_{\{s<t\}} \frac{\left|W_{s, t}^{(1)}\right|^{2 N}+\left|W(m)_{s, t}^{(1)}\right|^{2 N}}{|t-s|^{2 N \beta} .}
$$

By Lemma 3.5. we deduce that for any $0 \leq s<t \leq 1$,

$$
\begin{aligned}
\left|W_{s, t}^{(1)}-W(m)_{s, t}^{(1)}\right| \leq C A_{1}(m)^{\frac{1}{2 N}}|t-s|^{\frac{1}{p}} \\
\left|W_{s, t}^{(2)}-W(m)_{s, t}^{(2)}\right| \leq C\left[A_{2}(m)^{\frac{1}{2 M}}+A_{1}(m)^{\frac{1}{2 N}} \eta(m)^{\frac{1}{2 N}}\right]|t-s|^{\frac{2}{p}} .
\end{aligned}
$$


Finally, Schwarz's and Hölder's inequalities together with (2.34)-(2.37) conclude the proof of the theorem.

\section{Appendix}

Let $W^{H}=\left(W_{t}^{H}, t \in[0,1]\right)$ be a $d$-dimensional fractional Brownian motion with Hurst parameter $H \in] 0, \frac{1}{2}[\cup] \frac{1}{2}, 1[$ and integral representation given in (1.1).

Assume $H \in] \frac{1}{2}, 1$; by computing the integral of the right hand-side of (1.3), we obtain the following expression for the kernel $K^{H}$ defined in (1.2):

$$
K^{H}(t, s)=c_{H}\left(H-\frac{1}{2}\right) s^{H-\frac{1}{2}} F_{2}\left(\frac{t}{s}\right),
$$

where for $z>1$,

$$
F_{2}(z)=\int_{0}^{z-1} u^{H-\frac{3}{2}}(u+1)^{H-\frac{1}{2}} d u
$$

From (1.2), it follows that

$$
\frac{\partial K^{H}}{\partial t}(t, s)=c_{H}\left(H-\frac{1}{2}\right)\left(\frac{s}{t}\right)^{\frac{1}{2}-H}(t-s)^{H-\frac{3}{2}} .
$$

holds for any $H \in] 0, \frac{1}{2}[\cup] \frac{1}{2}, 1[$ and $0<s<t<1$. Consequently, for $H \in$ ] $0, \frac{1}{2}[$,

$$
\left|\frac{\partial K^{H}}{\partial t}(t, s)\right| \leq C|t-s|^{H-\frac{3}{2}}
$$

The next Lemma collects some technical estimates on the kernel $K^{H}(t, s)$.

Lemma 3.1. Let $0<s<t<1$.

(1) Assume $H \in] 0, \frac{1}{2}[$. Then,

$$
\begin{aligned}
& \left|K^{H}(t, s)\right| \leq C\left(s^{H-\frac{1}{2}} \mathbf{1}_{] 0, \frac{t}{2}[}(s)+(t-s)^{H-\frac{1}{2}} \mathbf{1}_{\left[\frac{t}{2}, t[\right.}(s)\right), \\
& \left|\frac{\partial K^{H}}{\partial s}(t, s)\right| \leq C\left(s^{H-\frac{3}{2}} \mathbf{1}_{] 0, \frac{t}{2}[}(s)+(t-s)^{H-\frac{3}{2}} \mathbf{1}_{\left[\frac{t}{2}, t[\right.}(s)\right), \\
& \left|\frac{\partial^{2} K^{H}}{\partial t \partial s}(t, s)\right| \leq C(t-s)^{H-\frac{3}{2}}\left(s^{-1} \mathbf{1}_{] 0, \frac{t}{2}[}(s)+(t-s)^{-1} \mathbf{1}_{\left[\frac{t}{2}, t[\right.}(s)\right) .
\end{aligned}
$$

(2) For $H \in] \frac{1}{2}, 1[$,

$$
\begin{aligned}
& \left|K^{H}(t, s)\right| \leq C\left((t-s)^{H-\frac{1}{2}} \mathbf{1}_{] 0, \frac{t}{2}[}(s)+s^{H-\frac{1}{2}} \mathbf{1}_{\left[\frac{t}{2}, t[\right.}(s)\right) \\
& \left|\frac{\partial K^{H}}{\partial s}(t, s)\right| \leq C(t-s)^{2 H-1}\left(s^{-\left(H+\frac{1}{2}\right)} \mathbf{1}_{] 0, \frac{t}{2}[}(s)+(t-s)^{-\left(H+\frac{1}{2}\right)} \mathbf{1}_{\left[\frac{t}{2}, t[\right.}(s)\right) .
\end{aligned}
$$


Proof. Assume first $H \in] 0, \frac{1}{2}[$. It is easy to check that, for any $u>0$,

$$
0<1-(u+1)^{H-\frac{1}{2}} \leq\left(\left(\frac{1}{2}-H\right) u\right) \wedge 1 .
$$

Hence, for $0<s<t, 0<u<\frac{t}{s}-1$,

$$
\begin{aligned}
u^{H-\frac{3}{2}}\left(1-(u+1)^{H-\frac{1}{2}}\right) \leq & C u^{H-\frac{1}{2}} \mathbf{1}_{] 0,1 \wedge\left(\frac{t}{s}-1\right)[}(u) \\
& +C u^{H-\frac{3}{2}} \mathbf{1}_{] 1 \wedge\left(\frac{t}{s}-1\right), \frac{t}{s}-1[}(u) .
\end{aligned}
$$

Thus, from (1.3), (3.10), it follows that

$$
\left|F_{1}\left(\frac{t}{s}\right)\right| \leq C \int_{0}^{\frac{t}{s}-1} u^{H-\frac{1}{2}} d u \leq C,
$$

for $\frac{t}{2} \leq s<t$, while for $0<s<\frac{t}{2}$,

$$
\left|F_{1}\left(\frac{t}{s}\right)\right| \leq C \int_{0}^{1} u^{H-\frac{1}{2}} d u+C \int_{1}^{\infty} u^{H-\frac{3}{2}} d u \leq C .
$$

Consequently

$$
\sup _{0 \leq s<t}\left|F_{1}\left(\frac{t}{s}\right)\right| \leq C
$$

and the identity (1.2) yields (3.5).

By differentiating with respect to the variable $s$ in (1.2) and using (3.11), we obtain

$$
\left|\frac{\partial K^{H}}{\partial s}(t, s)\right| \leq C\left(|t-s|^{H-\frac{3}{2}}+s^{H-\frac{3}{2}}+s^{-1} t|t-s|^{H-\frac{3}{2}}\right),
$$

which yields (3.6). The inequality (3.7) follows by differentiating with respect to the variable $s$ in (3.3).

Suppose now $H \in] \frac{1}{2}, 1$. Consider the function $F_{2}$ given in (3.2). Clearly, if $\frac{t}{s}-1 \leq 1$, that is, if $\frac{t}{2} \leq s<t$,

$$
\left|F_{2}\left(\frac{t}{s}\right)\right| \leq C
$$

Assume $\frac{t}{s}-1>1$. For any $\left.u \in\right] 1, \frac{t}{s}-1\left[,(1+u)^{H-\frac{1}{2}} \leq C u^{H-\frac{1}{2}}\right.$. Consequently,

$$
\left|F_{2}\left(\frac{t}{s}\right)\right| \leq C\left(\int_{0}^{1} u^{H-\frac{3}{2}} d u+\int_{1}^{\frac{t}{s}-1} u^{2 H-2} d u\right) \leq C\left(\frac{t}{s}\right)^{2 H-1} .
$$

The previous upper bounds, together with the representation of the kernel $K^{H}$ given in (3.1), imply

$$
\begin{aligned}
\left|K^{H}(t, s)\right| & \leq C\left(s^{H-\frac{1}{2}}\left(\frac{t}{s}\right)^{2 H-1} \mathbf{1}_{] 0, \frac{t}{2}[}(s)+s^{H-\frac{1}{2}} \mathbf{1}_{\left[\frac{t}{2}, t[\right.}(s)\right) \\
& \leq\left(s^{H-\frac{1}{2}} \mathbf{1}_{] 0, \frac{t}{2}[}(s)+s^{-H+\frac{1}{2}}(t-s)^{2 H-1} \mathbf{1}_{] 0, \frac{t}{2}[}(s)+s^{H-\frac{1}{2}} \mathbf{1}_{\left[\frac{t}{2}, t[\right.}(s)\right)
\end{aligned}
$$


and (3.8) follows.

Differentiating with respect to the variable $s$ in (3.1) yields

$$
\begin{aligned}
\left|\frac{\partial K^{H}}{\partial s}(t, s)\right| \leq & C\left(s^{H-\frac{3}{2}} F_{2}\left(\frac{t}{s}\right)+s^{H-\frac{1}{2}} \frac{t}{s^{2}}\left(\frac{t}{s}-1\right)^{H-\frac{3}{2}}\left(\frac{t}{s}\right)^{H-\frac{1}{2}}\right) \\
\leq & C\left(s^{H-\frac{3}{2}}\left(\frac{t}{s}\right)^{2 H-1} \mathbf{1}_{] 0, \frac{t}{2}[}(s)+s^{-\left(H+\frac{1}{2}\right)} t^{H+\frac{1}{2}}(t-s)^{H-\frac{3}{2}}\right. \\
& \left.+s^{H-\frac{3}{2}} \mathbf{1}_{\left[\frac{t}{2}, t[\right.}(s)\right),
\end{aligned}
$$

where in the last inequality we have applied the upper bounds for $F_{2}$ obtained before. Replacing in the last expression $t^{2 H-1}$ by $C\left(s^{2 H-1}+(t-\right.$ $\left.s)^{2 H-1}\right)$ and $t^{H+\frac{1}{2}}$ by $C\left(s^{H+\frac{1}{2}}+(t-s)^{H+\frac{1}{2}}\right)$, respectively, yields

$$
\left|\frac{\partial K^{H}}{\partial s}(t, s)\right| \leq C\left(s^{H-\frac{3}{2}}+(t-s)^{H-\frac{3}{2}}+s^{-\left(H+\frac{1}{2}\right)}(t-s)^{2 H-1}\right) .
$$

If $0<s<\frac{t}{2}$ then, $s<t-s$ and $(t-s)^{H-\frac{3}{2}}<s^{H-\frac{3}{2}}<s^{-\left(H+\frac{1}{2}\right)}(t-s)^{2 H-1}$, while for $\frac{t}{2} \leq s<t$, the previous inequalities are reversed accordingly. Hence (3.9) clearly follows from (3.12).

We introduce the notation

$$
\underline{t}_{m}=\left[2^{m} t\right] 2^{-m} \text { and } \quad \bar{t}_{m}=\underline{t}_{m}+2^{-m},
$$

for any $m \in \mathbb{N}$. Notice that, $K_{m}^{H}$ given in (1.5) satisfies $K_{m}^{H}(t, s)=0$ if $s \geq \bar{t}_{m}$.

In the next result, we give a bound for the approximation in quadratic mean of the kernel $K^{H}$ by its projection $K_{m}^{H}$.

Lemma 3.2. (1) Let $H \in] 0, \frac{1}{2}[\cup] \frac{1}{2}, 1[$. There exists a positive constant $C$ such that for any $0<s<t \leq 1$,

$\sup _{m \geq 1} \int_{0}^{1}\left(\left|K_{m}^{H}(t, u)-K_{m}^{H}(s, u)\right|^{2}+\left|K^{H}(t, u)-K^{H}(s, u)\right|^{2}\right) d u \leq C|t-s|^{2 H}$.

(2) For $H \in] 0, \frac{1}{2}[$,

$$
\int_{0}^{1}\left|K^{H}(t, u)-K_{m}^{H}(t, u)\right|^{2} d u \leq C\left(t \wedge 2^{-m}\right)^{2 H} .
$$

(3) For $H \in] \frac{1}{2}, 1[$ and any $\lambda \in] 0, \frac{1}{2 H+1}[$,

$$
\int_{0}^{1}\left|K^{H}(t, u)-K_{m}^{H}(t, u)\right|^{2} d u \leq C 2^{-2 m \lambda} t^{2(H-\lambda)} .
$$

Proof. The operator $\pi_{m}$ is a contraction on $L^{2}[0,1]$. Thus,

$$
\sup _{m \geq 1} \int_{0}^{1}\left(\left|K_{m}^{H}(t, u)-K_{m}^{H}(s, u)\right|^{2}+\left|K^{H}(t, u)-K^{H}(s, u)\right|^{2}\right) d u
$$




$$
\leq 2 \int_{0}^{1}\left|K^{H}(t, u)-K^{H}(s, u)\right|^{2} d u=2 E\left(\left|W_{t}^{H}-W_{s}^{H}\right|^{2}\right)=2|t-s|^{2 H},
$$

proving(3.14).

By the same argument,

$$
\int_{0}^{1}\left|K^{H}(t, u)-K_{m}^{H}(t, u)\right|^{2} d u \leq 4 \int_{0}^{1}\left|K^{H}(t, u)\right|^{2} d u=4 t^{2 H} .
$$

Therefore (3.15) holds for $t \leq C 2^{-m}$.

Fix $t \in \Delta_{I}^{m}$ with $I>7$. We assume first $\left.H \in\right] 0, \frac{1}{2}[$. Consider the decomposition

$$
\int_{0}^{1}\left|K^{H}(t, u)-K_{m}^{H}(t, u)\right|^{2} d u \leq C \sum_{i=1}^{5} T_{i}(t)
$$

with

$$
\begin{aligned}
& T_{1}(t)=\int_{0}^{t_{2}^{m}}\left|K^{H}(t, u)-K_{m}^{H}(t, u)\right|^{2} d u, \\
& T_{2}(t)=\int_{t_{I-3}^{m}}^{t_{I}^{m}}\left|K^{H}(t, u)-K_{m}^{H}(t, u)\right|^{2} d u, \\
& T_{3}(t)=\sum_{k=3}^{\left[2^{m-1} t\right]} \int_{\Delta_{k}^{m}}\left|K^{H}(t, u)-K_{m}^{H}(t, u)\right|^{2} d u, \\
& T_{4}(t)=\sum_{k=\left[2^{m-1} t\right]+2}^{I-3} \int_{\Delta_{k}^{m}}\left|K^{H}(t, u)-K_{m}^{H}(t, u)\right|^{2} d u, \\
& T_{5}(t)=\int_{\left.\Delta_{\left[2^{m-1}\right.}^{m} t\right]+1}\left|K^{H}(t, u)-K_{m}^{H}(t, u)\right|^{2} d u .
\end{aligned}
$$

Schwarz's inequality and (3.5) imply

$$
T_{1}(t) \leq 4 \int_{0}^{t_{2}^{m}}\left|K^{H}(t, u)\right|^{2} d u \leq C \int_{0}^{t_{2}^{m}} u^{2 H-1} d u=C 2^{-2 m H} .
$$

Similarly,

$$
T_{2}(t) \leq 4 \int_{t_{I-3}^{m}}^{t_{I}^{m}}\left|K^{H}(t, u)\right|^{2} d u \leq C \int_{t_{I-3}^{m}}^{t}|t-u|^{2 H-1} d u=C 2^{-2 m H} .
$$

Let $\lambda \in] H, 1\left[\right.$ and $k=3, \ldots,\left[2^{m-1} t\right]$, which implies $\left.\Delta_{k}^{m} \subset\right] 0, \frac{t}{2}[$. By Schwarz's inequality, the mean value theorem and (3.5), (3.6), we obtain

$$
\begin{gathered}
\int_{\Delta_{k}^{m}}\left|K^{H}(t, u)-K_{m}^{H}(t, u)\right|^{2} d u \leq 2^{m} \int_{\Delta_{k}^{m}} d u \int_{\Delta_{k}^{m}} d v\left|K^{H}(t, u)-K^{H}(t, v)\right|^{2} \\
\leq 2^{m} \int_{\Delta_{k}^{m}} d u \int_{\Delta_{k}^{m}} d v\left|K^{H}(t, u)-K^{H}(t, v)\right|^{2 \lambda}|| K^{H}(t, u)|+| K^{H}(t, v)||^{2(1-\lambda)}
\end{gathered}
$$




$$
\leq C 2^{-m(2 \lambda-1)} \int_{\Delta_{k}^{m}} d u \int_{\Delta_{k}^{m}} d v\left((u \wedge v)^{2 H-1-2 \lambda}\right) .
$$

For $u, v \in \Delta_{k}^{m}, u \wedge v \geq u-2^{-m}$; thus,

$$
T_{3}(t) \leq C 2^{-2 m \lambda} \int_{t_{2}^{m}}^{t_{\left[2^{m-1} t\right]}^{m}} d u\left(u-2^{-m}\right)^{2 H-1-2 \lambda} \leq C 2^{-2 m H} .
$$

Fix now $k=\left[2^{m-1} t\right]+2, \ldots, I-3$, so that $\Delta_{k}^{m} \subset\left[\frac{t}{2}, t[\right.$. In this case

$$
\begin{gathered}
\int_{\Delta_{k}^{m}}\left|K^{H}(t, u)-K_{m}^{H}(t, u)\right|^{2} d u \leq C 2^{-m(2 \lambda-1)} \\
\times \int_{\Delta_{k}^{m}} d u \int_{\Delta_{k}^{m}} d v(t-(u \vee v))^{2 H-1-2 \lambda}
\end{gathered}
$$

Since for $u, v \in \Delta_{k}^{m}, t-(u \vee v) \geq t-u-2^{-m} \geq t_{I-2}^{m}-u$, the previous estimate implies

$$
T_{4}(t) \leq C 2^{-2 m \lambda} \int_{t_{\left[2^{m-1} t\right]}^{m}}^{t_{I-3}^{m}} d u\left(t_{I-2}^{m}-u\right)^{2 H-1-2 \lambda} \leq C 2^{-2 m H} .
$$

We study the term $T_{5}(t)$ using the same method as for $T_{3}(t), T_{4}(t)$, as follows:

$$
\begin{aligned}
T_{5}(t) \leq & 2^{m} \int_{\Delta_{\left[2^{m-1} t\right]+1}^{m}} d u \int_{\Delta_{\left[2^{m-1} t\right]+1}^{m}} d v\left|K^{H}(t, u)-K^{H}(t, v)\right|^{2} \\
\leq & C 2^{-m(2 \lambda-1)} \int_{\Delta_{\left[2^{m-1} t\right]+1}^{m}} ! d u \int_{\Delta_{\left[2^{m-1} t\right]+1}^{m}} d v\left((u \wedge v)^{H-\frac{3}{2}}+\left(t-(u \vee v)^{H-\frac{3}{2}}\right)^{2 \lambda}\right. \\
& \times\left((u \wedge v)^{H-\frac{1}{2}}+\left(t-(u \vee v)^{H-\frac{1}{2}}\right)^{2(1-\lambda)} .\right.
\end{aligned}
$$

For $u, v \in \Delta_{\left[2^{m-1} t\right]+1}^{m}, u \wedge v>\frac{t}{2}-2^{-m}, u \vee v<\frac{t}{2}+2^{-m}$ and $t-(u \vee v)>$ $\frac{t}{2}-2^{-m}$. Thus, the last integral is bounded by

$$
\int_{\left.\Delta_{\left[2^{m-1}\right.}^{m} t\right]+1} d u \int_{\Delta_{\left[2^{m-1} t\right]+1}^{m}} d v\left(\frac{t}{2}-2^{-m}\right)^{2 H-1-2 \lambda} .
$$

Moreover, since we are assuming that $t \in \Delta_{I}^{m}$, with $I>7, \frac{t}{2}-2^{-m} \geq 2^{-m+1}$. Thus, we finally obtain for $\lambda=\frac{1}{2}$,

$$
T_{5}(t) \leq C 2^{-2 m H} \text {. }
$$

Then (3.15) follows from the upper bounds obtained so far for $T_{i}(t), i=$ $1, \ldots, 5$.

Notice that we have also proved that for $H \in] 0, \frac{1}{2}[$,

$$
\sum_{k=3}^{I-3} 2^{m} \int_{\Delta_{k}^{m}} d u \int_{\Delta_{k}^{m}} d v\left|K^{H}(t, u)-K^{H}(t, v)\right|^{2} \leq C 2^{-2 m H} .
$$


Assume now $H \in] \frac{1}{2}, 1[$ and fix $\lambda \in] 0, \frac{1}{2 H+1}[$, so that $H-\lambda>0$. Since the inequality (3.17) holds for any $H \in] 0, \frac{1}{2}[\cap] \frac{1}{2}, 1[$, (3.16) holds for any $t \leq C 2^{-m}$. Let now $t \in \Delta_{I}^{m}$, with $I>7$. We apply a similar method as we used in the case $H \in] 0, \frac{1}{2}$ [, using the decomposition (3.18). In fact, owing to (3.8),

$$
\begin{aligned}
& T_{1}(t) \leq C \int_{0}^{t_{2}^{m}}(t-u)^{2 H-1} d u \leq C 2^{-m} t^{2 H-1}, \\
& T_{2}(t) \leq C \int_{t_{I-3}^{m}}^{t_{I}^{m}} u^{2 H-1} d u \leq C 2^{-m} t^{2 H-1} .
\end{aligned}
$$

Fix $k=3, \ldots,\left[2^{m-1} t\right]$. Schwarz's inequality, along with the mean value theorem and (3.8), (3.9), imply

$$
\begin{gathered}
\int_{\Delta_{k}^{m}}\left|K^{H}(t, u)-K_{m}^{H}(t, u)\right|^{2} d u \leq 2^{m} \int_{\Delta_{k}^{m}} d u \int_{\Delta_{k}^{m}} d v\left|K^{H}(t, u)-K^{H}(t, v)\right|^{2 \lambda} \\
\times\left.|| K^{H}(t, u)|+| K^{H}(t, v)\right|^{2(1-\lambda)} \\
\leq C 2^{-m(2 \lambda-1)} \int_{\Delta_{k}^{m}} d u \int_{\Delta_{k}^{m}} d v\left((t-(u \wedge v))^{(\lambda+1)(2 H-1)}(u \wedge v)^{-\lambda(2 H+1)}\right. \\
\leq C 2^{-2 m \lambda} t^{(\lambda+1)(2 H-1)} \int_{\Delta_{k}^{m}} d u\left(u-2^{-m}\right)^{-\lambda(2 H+1)} .
\end{gathered}
$$

Since $\lambda<\frac{1}{2 H+1}$, we have

$$
T_{3}(t) \leq C 2^{-2 m \lambda} t^{2(H-\lambda)} .
$$

Let now $k=\left[2^{m-1} t\right]+2, \ldots, I-3$. With similar arguments as before, we deduce

$$
\begin{gathered}
\int_{\Delta_{k}^{m}}\left|K^{H}(t, u)-K_{m}^{H}(t, u)\right|^{2} d u \leq 2^{m} \int_{\Delta_{k}^{m}} d u \int_{\Delta_{k}^{m}} d v\left|K^{H}(t, u)-K^{H}(t, v)\right|^{2 \lambda} \\
\times\left.|| K^{H}(t, u)|+| K^{H}(t, v)\right|^{2(1-\lambda)} \\
\leq C 2^{-m(2 \lambda-1)} \int_{\Delta_{k}^{m}} d u \int_{\Delta_{k}^{m}} d v(t-(u \vee v))^{\lambda(2 H-3)}(u \vee v)^{(1-\lambda)(2 H-1)} \\
\leq C 2^{-2 m \lambda} t^{(1-\lambda)(2 H-1)} \int_{\Delta_{k}^{m}} d u\left(t-u-2^{-m}\right)^{\lambda(2 H-3)} .
\end{gathered}
$$

For $\lambda<\frac{1}{2 H+1}, \lambda(2 H-3)+1>0$. Hence,

$$
T_{4}(t) \leq C 2^{-2 m \lambda} t^{(1-\lambda)(2 H-1)} \int_{\frac{t}{2}}^{t_{I-3}^{m}}\left(t-u-2^{-m}\right)^{\lambda(2 H-3)} \leq C 2^{-2 m \lambda} t^{2(H-\lambda)} .
$$

Finally, we study the contribution of $T_{5}(t)$ as follows.

$$
T_{5}(t) \leq 2^{m} \int_{\Delta_{\left[2^{m-1} t\right]+1}^{m}} d u \int_{\Delta_{\left[2^{m-1} t\right]+1}^{m}} d v\left|K^{H}(t, u)-K^{H}(t, v)\right|^{2}
$$




$$
\begin{aligned}
\leq C 2^{-m(2 \lambda-1)} \int_{\left.\Delta_{\left[2^{m}-1\right.}^{m} t\right]+1} d u \int_{\Delta_{\left[2^{m-1} t\right]+1}^{m}} d v\left((t-(u \wedge v))^{2 H-1}\right. \\
\left.\quad \times\left((u \wedge v)^{-\left(H+\frac{1}{2}\right)}+(t-(u \vee v))^{-\left(H+\frac{1}{2}\right)}\right)\right)^{2 \lambda} \\
\quad \times\left((t-(u \wedge v))^{H-\frac{1}{2}}+(u \vee v)^{H-\frac{1}{2}}\right)^{2(1-\lambda)} .
\end{aligned}
$$

For $u, v \in \Delta_{\left[2^{m-1} t\right]+1}^{m}, u \wedge v>C_{1} t, u \vee v<C_{2} t, t-(u \wedge v)<C_{3} t$ and $t-(u \vee v)>C_{4} t$. Thus,

$$
T_{5}(t) \leq C 2^{-m(2 \lambda-1)} 2^{-2 m} t^{2(H-\lambda)-1} \leq C 2^{-2 m \lambda} t^{2(H-\lambda)}
$$

The estimates obtained so far imply (3.16).

In the next Lemma we prove a simple extension of a well-known integration formula for bounded variation functions.

Lemma 3.3. For any $h \in \mathcal{H}, t \geq 0$,

$$
\int_{0}^{t} h(u) h(d u)=\frac{h^{2}(t)}{2}
$$

where the integral is understood in the sense of Proposition 5 in [7].

Proof. Let $n \geq 1$ and let $h(n)$ be the function obtained by linear interpolation on the $n$-th dyadic grid of $h$. We have proved in [7], Theorem 9 that

$$
\lim _{n \rightarrow \infty} \int_{0}^{t} h(n)(u) h(n)(d u)=\int_{0}^{t} h(u) h(d u),
$$

for any $t \geq 0$. Since (3.20) is true with $h$ replaced by $h(n)$, the result follows.

The following result gives an upper bound for the $L^{2}$ norm of a Skorohod integral of a Gaussian process.

Lemma 3.4. Let $X_{t}=\int_{0}^{1} g(t, s) d B_{s}, t \in[0,1]$, with $g$ a deterministic function belonging to $L^{2}\left([0,1]^{2}\right)$. Then, the Skorohod integral $\int_{0}^{1} X_{s} d B_{s}$ satisfies

$$
E\left(\int_{0}^{1} X_{s} d B_{s}\right)^{2} \leq C \int_{0}^{1} d s \int_{0}^{1} d r|g(s, r)|^{2}
$$

Proof. The isometry property of the Skorohod integral ([], Equation (1.48)) yields

$$
E\left(\int_{0}^{1} X_{s} d B_{s}\right)^{2} \leq C \int_{0}^{1} E\left(X_{s}\right)^{2} d s+\int_{0}^{1} d s \int_{0}^{1} d r E\left(\left|D_{r} X_{s}\right|^{2}\right) .
$$

Since $E\left(X_{s}\right)^{2}=\int_{0}^{1}|g(s, r)|^{2} d r$ and the Malliavin derivative $D_{r} X_{s}$ is equal to $g(s, r)$, (3.21) follows. 
We conclude this section by proving an extension of the Garsia-RademichRumsey lemma used to estimate $\tilde{d}_{p}(X, Y)$ when $X$ and $Y$ are geometric rough paths with roughness $p \in[2, \infty$ [ (see [6], Definition 3.3.3).

Lemma 3.5. Let $X$ and $Y$ be geometric rough paths with the same roughness $p \in\left[2,+\infty\left[\right.\right.$. Set $k=[p]$. For $i=1, \cdots, k$, let $M_{i} \geq 1, \alpha_{i}=\frac{i}{p}+\frac{1}{M_{i}}$. Suppose that

$$
\begin{gathered}
\int_{0}^{1} \int_{0}^{1} d s d t 1_{\{s \leq t\}} \frac{\left|X_{s, t}^{(i)}\right|^{2 M_{i}}+\left|Y_{s, t}^{(i)}\right|^{2 M_{i}}}{|t-s|^{2 M_{i} \alpha_{i}}} \leq A_{i}, \quad 1 \leq i \leq k-1, \\
\int_{0}^{1} \int_{0}^{1} d s d t 1_{\{s \leq t\}} \frac{\left|X_{s, t}^{(i)}-Y_{s, t}^{(i)}\right|^{2 M_{i}}}{|t-s|^{2 M_{i} \alpha_{i}}} \leq B_{i}, \quad 1 \leq i \leq k .
\end{gathered}
$$

Then, there exists a constant $C>0$ such that for any $0 \leq s<t \leq 1$,

$$
\begin{array}{cl}
\left|X_{s, t}^{(i)}\right|+\left|Y_{s, t}^{(i)}\right| \leq C F_{i}|t-s|^{\frac{i}{p}}, & 1 \leq i \leq k-1, \\
\left|X_{s, t}^{(i)}-Y_{s, t}^{(i)}\right| \leq C G_{i}|t-s|^{\frac{i}{p}}, & 1 \leq i \leq k .
\end{array}
$$

where $F_{i}$ and $G_{i}$ are defined recursively by

$$
\begin{aligned}
F_{i} & =A_{i}^{\frac{1}{2 M_{i}}}+\sum_{j=1}^{i-1} F_{j} F_{i-j}, \quad 1 \leq i \leq k-1, \\
G_{i} & =B_{i}^{\frac{1}{2 M_{i}}}+\sum_{j=1}^{i-1} G_{j} F_{i-j}, \quad 1 \leq i \leq k .
\end{aligned}
$$

Remark: For rough paths $X, Y$ of roughness $p \in\left[1, \infty\left[, X_{s, t}^{(1)}-X_{s, t}^{(1)}=\right.\right.$ $(X-Y)_{s, t}^{(1)}$. The usual version of the Garsia-Rademich-Rumsey lemma yields the following. If

$$
\int_{0}^{1} \int_{0}^{1} d s d t 1_{\{s \leq t\}} \frac{\left|X_{s, t}^{(1)}-Y_{s, t}^{(1)}\right|^{2 M_{1}}}{|t-s|^{2 M_{1} \alpha_{1}}} \leq B_{1},
$$

then $\left|X_{s, t}^{(1)}-Y_{s, t}^{(1)}\right| \leq C B_{1}^{\frac{1}{2 M_{1}}}|t-s|^{\frac{1}{p}}$. Similarly, if

$$
\int_{0}^{1} \int_{0}^{1} d s d t 1_{\{s \leq t\}} \frac{\left|X_{s, t}^{(1)}\right|^{2 M_{1}}+\left|Y_{s, t}^{(1)}\right|^{2 M_{1}}}{|t-s|^{2 M_{1} \alpha_{1}}} \leq A_{1}
$$

then $\left|X_{s, t}^{(1)}\right|+\left|Y_{s, t}^{(1)}\right| \leq C A_{1}^{\frac{1}{2 M_{1}}}|t-s|^{\frac{1}{p}}$.

Proof of Lemma 3.5: Throughout the proof, the constants $F_{i}, 1 \leq i \leq k-1$ and $G_{i}, 1 \leq i \leq k$ are defined by (3.26), (3.27), respectively. We introduce the following assumption:

$\left(H_{i}\right)$

$$
\int_{0}^{1} \int_{0}^{1} d s d t 1_{\{s \leq t\}} \frac{\left|X_{s, t}^{(i)}-Y_{s, t}^{(i)}\right|^{2 M_{i}}}{|t-s|^{2 M_{i} \alpha_{i}}} \leq B_{i}
$$




$$
\begin{aligned}
& \left|X_{s, t}^{(j)}\right|+\left|Y_{s, t}^{(j)}\right| \leq C F_{j}|t-s|^{\frac{j}{p}}, \quad 1 \leq j \leq i-1, \\
& \left|X_{s, t}^{(j)}-Y_{s, t}^{(j)}\right| \leq C G_{j}|t-s|^{\frac{j}{p}}, \quad 1 \leq j \leq i-1,
\end{aligned}
$$

$i \in\{2, \ldots, k\}$, and we prove that $\left(H_{i}\right)$ implies

$$
\left|X_{s, t}^{(i)}-Y_{s, t}^{(i)}\right| \leq C G_{i}|t-s|^{\frac{i}{p}}
$$

For this, we use an argument similar to the proof of Theorem 2.1.3 in 9 .

Indeed, for every $t \in[0,1]$, set

$$
I(t)=\int_{0}^{t} \frac{\left|X_{s, t}^{(i)}-Y_{s, t}^{(i)}\right|^{2 M_{i}}}{|t-s|^{2 M_{i} \alpha_{i}}} d s, J(t)=\int_{t}^{1} \frac{\left|X_{t, u}^{(i)}-Y_{t, u}^{(i)}\right|^{2 M_{i}}}{|u-t|^{2 M_{i} \alpha_{i}}} d u .
$$

Then $\int_{0}^{1} I(t) d t=\int_{0}^{1} J(t) d t \leq B_{i}$ and there exists $t_{0}>0$ such that $I\left(t_{0}\right)+$ $J\left(t_{0}\right) \leq 2 A_{i}$. We construct by induction a decreasing sequence $\left(t_{n}, n \geq 0\right)$ such that $\lim _{n} t_{n}=0$ and an increasing sequence $\left(s_{n}, n \geq 0\right)$ such that $s_{0}=t_{0}, \lim _{n} s_{n}=1$, and such that there exists $C>0$ such that for every $n \geq 1$,

$$
\begin{gathered}
\left|X_{t_{n}, t_{0}}^{(i)}-Y_{t_{n}, t_{0}}^{(i)}\right| \leq C \int_{0}^{1}\left|8 B_{i}\right|^{\frac{1}{2 M_{i}}} u^{\frac{i}{p}-1} d u+C \sum_{j=1}^{i-1} F_{j} G_{i-j}, \\
\left|X_{s_{0}, s_{n}}^{(i)}-Y_{s_{0}, s_{n}}^{(i)}\right| \leq C \int_{0}^{1}\left|8 B_{i}\right|^{\frac{1}{2 M_{i}}} u^{\frac{i}{p}-1} d u+C \sum_{j=1}^{i-1} F_{j} G_{i-j} .
\end{gathered}
$$

Then Chen's identity implies as $n \rightarrow+\infty$,

$$
\begin{aligned}
& \left|X_{0,1}^{(i)}-Y_{0,1}^{(i)}\right| \leq\left|X_{0, t_{0}}^{(i)}-Y_{0, t_{0}}^{(i)}\right|+\left|X_{t_{0}, 1}^{(i)}-Y_{t_{0}, 1}^{(i)}\right| \\
& \quad+\sum_{j=1}^{i-1}\left(\left|X_{0, t_{0}}^{(j)}-Y_{0, t_{0}}^{(j)}\right|\left|X_{t_{0}, 1}^{(i-j)}\right|+\left|Y_{0, t_{0}}^{(j)}\right|\left|X_{t_{0}, 1}^{(i-j)}-Y_{t_{0}, 1}^{(i-j)}\right|\right) .
\end{aligned}
$$

With the hypothesis $\left(H_{i}\right)$, we obtain (3.28) with $s=0$ and $t=1$.

To construct $\left(t_{n}\right)$, we suppose that $t_{n-1}$ has been chosen. Let $d_{n-1}$ be defined by $d_{n-1}^{\alpha_{i}}=\frac{1}{2} t_{n-1}^{\alpha_{i}}$. Then there exists $\left.t_{n} \in\right] 0, d_{n-1}[$ such that

$$
I\left(t_{n}\right) \leq \frac{4 B_{i}}{d_{n-1}} \quad \text { and } \quad \frac{\left|X_{t_{n}, t_{n-1}}^{(i)}-Y_{t_{n}, t_{n-1}}^{(i)}\right|^{2 M_{i}}}{\left|t_{n-1}-t_{n}\right|^{2 M_{i} \alpha_{i}}} \leq \frac{2 I\left(t_{n-1}\right)}{d_{n-1}} .
$$

Indeed, the sets where each one of these inequalities may fail has Lebesgue measure less that $\frac{d_{n-1}}{2}$. Furthermore, for every $n \geq 0,2 d_{n+1}^{\alpha_{i}}=t_{n+1}^{\alpha_{i}} \leq$ $d_{n}^{\alpha_{i}}=\frac{1}{2} t_{n}^{\alpha_{i}}$ and $\left|t_{n}-t_{n+1}\right|^{\alpha_{i}} \leq t_{n}^{\alpha_{i}}=2 d_{n}^{\alpha_{i}} \leq 4\left(d_{n}^{\alpha_{i}}-d_{n+1}^{\alpha_{i}}\right)$. Hence there exists $a \in] 0,1\left[\right.$ such that $t_{n+1} \leq a t_{n}$, so that $\lim _{n} t_{n}=0$ and more precisely,

while for any $n \geq 1$,

$$
t_{n} \leq a^{n} t_{0}
$$

$$
\left|X_{t_{n+1}, t_{n}}^{(i)}-Y_{t_{n+1}, t_{n}}^{(i)}\right| \leq\left|2 I\left(t_{n}\right)\right|^{\frac{1}{2 M_{i}}} d_{n}^{-\frac{1}{2 M_{i}}}\left|t_{n}-t_{n+1}\right|^{\alpha_{i}}
$$




$$
\begin{aligned}
& \leq\left|8 B_{i}\right|^{\frac{1}{2 M_{i}}}\left|d_{n} d_{n-1}\right|^{-\frac{1}{2 M_{i}}} 4\left|d_{n}^{\alpha_{i}}-d_{n+1}^{\alpha_{i}}\right| \\
& \leq 4 \alpha_{i} \int_{d_{n+1}}^{d_{n}}\left|8 B_{i}\right|^{\frac{1}{2 M_{i}}} u^{-\frac{1}{M_{i}}+\alpha_{i}-1} d u .
\end{aligned}
$$

Let $b=a^{\frac{1}{p}}<1$; Chen's identity, $\left(H_{i}\right)$ and (3.33) imply that for any $n \geq 1$,

$$
\begin{aligned}
& \left|X_{t_{n+1}, t_{0}}^{(i)}-Y_{t_{n+1}, t_{0}}^{(i)}\right| \leq\left|X_{t_{n}, t_{0}}^{(i)}-Y_{t_{n}, t_{0}}^{(i)}\right|+\left|X_{t_{n+1}, t_{n}}^{(i)}-Y_{t_{n+1}, t_{n}}^{(i)}\right| \\
& \quad+\sum_{j=1}^{i-1}\left(\left|X_{t_{n+1}, t_{n}}^{(j)}-Y_{t_{n+1}, t_{n}}^{(j)}\right|\left|X_{t_{n}, t_{0}}^{(i-j)}\right|+\left|Y_{t_{n+1}, t_{n}}^{(j)}\right|\left|X_{t_{n}, t_{0}}^{(i-j)}-Y_{t_{n}, t_{0}}^{(i-j)}\right|\right) \\
& \leq\left|X_{t_{n}, t_{0}}^{(i)}-Y_{t_{n}, t_{0}}^{(i)}\right|+C \int_{d_{n+1}}^{d_{n}}\left|8 B_{i}\right|^{\frac{1}{2 M_{i}}} u^{\frac{i}{p}-1} d u \\
& \quad+C \sum_{j=1}^{i-1}\left(G_{j} F_{i-j}+F_{j} G_{i-j}\right)\left|t_{n}-t_{n+1}\right|^{\frac{j}{p}}\left|t_{0}-t_{n}\right|^{\frac{i-j}{p}}
\end{aligned}
$$

Since $\sup _{1 \leq j \leq i-1}\left|t_{n}-t_{n+1}\right|^{\frac{j}{p}} \leq t_{n}^{\frac{1}{p}} \leq C b^{n}<1$, an easy induction on $n$ implies that for any $n \geq 1$,

$$
\left|X_{t_{n}, t_{0}}^{(i)}-Y_{t_{n}, t_{0}}^{(i)}\right| \leq C \int_{0}^{1}\left|8 B_{i}\right|^{\frac{1}{2 M_{i}}} u^{\frac{i}{p}-1} d u+C\left(\sum_{j=1}^{i-1} G_{j} F_{i-j}\right)\left(\sum_{l=0}^{n-2} b^{l}\right),
$$

which implies (3.29). To prove (3.30), we proceed in a similar way, exchanging the endpoints of the interval $[0,1]$. Recall that $s_{0}=t_{0}$; suppose that $s_{n-1}$ has been defined and let $\delta_{n-1}$ be such that $\left|1-\delta_{n-1}\right|^{\alpha_{i}}=\frac{1}{2}\left|1-s_{n-1}\right|^{\alpha_{i}}$. There exists $\left.s_{n} \in\right] \delta_{n-1}, 1[$ such that

$$
J\left(s_{n}\right) \leq \frac{4 B_{i}}{1-\delta_{n-1}} \quad \text { and } \quad \frac{\left|X_{s_{n-1}, s_{n}}^{(i)} Y_{s_{n-1}, s_{n}}^{(i)}\right|^{2 M_{i}}}{\left|s_{n}-s_{n-1}\right|^{\alpha_{i}}} \leq \frac{2 J\left(s_{n-1}\right)}{1-\delta_{n-1}} .
$$

Then for every $n \geq 1,2\left|1-\delta_{n+1}\right|^{\alpha_{i}}=\left|1-s_{n+1}\right|^{\alpha_{i}} \leq\left|1-\delta_{n}\right|^{\alpha_{i}}=\frac{1}{2}\left|1-t_{n}\right|^{\alpha_{i}}$, so that $s_{n} \leq \delta_{n} \leq s_{n+1} \leq \delta_{n+1}$ and for some $\left.\bar{a} \in\right] 0,1[$

$$
1-s_{n} \leq \bar{a}^{n}\left(1-t_{0}\right)
$$

so that $\lim _{n} s_{n}=1$ and computations similar to those proving (3.33) yield

$$
\left|X_{s_{n}, s_{n+1}}^{(i)}-Y_{s_{n}, s_{n+1}}^{(i)}\right| \leq 4 \alpha_{i} \int_{\delta_{n}}^{\delta_{n+1}}\left|8 B_{i}\right|^{\frac{1}{2 M_{i}}} u^{-\frac{1}{M_{i}}+\alpha_{i}-1} d u .
$$

Thus if $\bar{b}=\bar{a}^{\frac{1}{p}}<1$, Chen's identity and $\left(H_{i}\right)$ imply

$$
\left|X_{t_{0}, s_{n}}^{(i)}-Y_{t_{0}, s_{n}}^{(i)}\right| \leq C \int_{t_{0}}^{s_{n}}\left|8 B_{i}\right|^{\frac{1}{2 M_{i}}} u^{\frac{i}{p}-1} d u+C\left(\sum_{j=1}^{i-1} F_{j} G_{i-j}\right)\left(\sum_{l=0}^{n-1} \bar{b}^{l}\right),
$$

which completes the proof of (3.30) and hence that of (3.28) for $s=0, t=1$. 
To deduce (3.28), for any $s, t \in[0,1]$ with $s<t$, define $\bar{X}_{u}=X_{s+(t-s) u}$, $\bar{Y}_{u}=Y_{s+(t-s) u}$ for $u \in[0,1]$. Then $\bar{X}$ and $\bar{Y}$ are geometric rough paths with the same roughness $p$. Moreover, for $0 \leq u<v \leq 1, j=1, \cdots, k$, $\bar{X}_{u, v}^{(j)}=X_{s+(t-s) u, s+(t-s) v}^{(j)}$. In fact, by a change of variables, we see that this identity is obvious for smooth rough paths and therefore it is trivially extended to geometric rough paths.

Furthermore,

$$
\begin{aligned}
\int_{0}^{1} \int_{0}^{1} d u d v 1_{\{u<v\}} \frac{\left|\bar{X}_{u, v}^{(i)}-\bar{Y}_{u, v}^{(i)}\right|^{2 M_{i}}}{|v-u|^{2 M_{i} \alpha_{i}}} \\
=(t-s)^{-2+2 \alpha_{i} M_{i}} \int_{s}^{t} \int_{s}^{t} d u d v 1_{\{u<v\}} \frac{\left|X_{u, v}^{(i)}-Y_{u, v}^{(i)}\right|^{2 M_{i}}}{|v-u|^{2 M_{i} \alpha_{i}}} \\
\leq(t-s)^{-2+2 \alpha_{i} M_{i}} B_{i}=(t-s)^{2 M_{i} \frac{i}{p}} B_{i} .
\end{aligned}
$$

Hence, if the pair $(X, Y)$ satisfies $\left(H_{i}\right)$ then $(\bar{X}, \bar{Y})$ satisfies a similar property with constants $\bar{A}_{j}=(t-s)^{2 M_{j} \frac{j}{p}} A_{j}, \bar{F}_{j}=|t-s|^{\frac{j}{p}} F_{j}, 1 \leq j \leq i-1$, $\bar{B}_{j}=(t-s)^{2 M_{j} \frac{j}{p}} B_{j}, \bar{G}_{j}=|t-s|^{\frac{j}{p}}, 1 \leq j \leq i$. This finishes the proof of (3.28).

Taking in the preceding arguments first $X \equiv 0$ and then $Y \equiv 0$, we see recursively that (3.22) implies $\left(H_{i}\right)$ for any $i=1, \ldots, k-1$, with $B_{i}=A_{i}$. Hence we obtain (3.24). Moreover, we also see that $\left(H_{i}\right)$ holds true for any $i=1, \ldots, k$, whenever (3.22), (3.23) are satisfied. This concludes the proof.

Acknowledgments The first named author whishes to thank the Centre de Recerca Matemàtica in Bellaterra and the Universitat de Barcelona for their support and hospitality in the fall of 2004, when discussions on the content of this paper started.

\section{REFERENCES}

[1] E. Alòs, O. Mazet, D. Nualart: Stochastic calculus with respect to Gaussian processes. The Annals of Probab. Vol. 29, No. 2, 766-801 (2001).

[2] L. Coutin, Z. Qian: Stochastic analysis, rough path analysis and fractional Brownian motions. Probab. Theory Relat. Fields 122, 108-140 (2002).

[3] L. Coutin, P. Friz, N. Victoir: Good rough path sequences and applications to anticipating and fractional stochastic calculus. arXiv:math.PR/0501197 January 2005.

[4] L. Decreusefond, S. Üstünel: Stochastic analysis of the fractional Brownian motion. Potential Analysis 10, 177-214 (1999).

[5] P. Friz, N. Victoir: A note on the notion of geometric rough paths. arXiv:math.FA/0403115 March 2004.

[6] T. Lyons, Z. Qian: System Control and Rough Paths. Oxford Mathematical Monographs. Oxford Science Publiccations. Clarendon Press, Oxford 2002.

[7] A. Millet, M. Sanz-Solé: Large deviations for rough paths of the fractional Brownian motion. Annales de l'Institut Poincaré (to appear), arXiv:math.PR/0412200), December 2004. 
[8] D. Nualart: The Malliavin Calculus and Related Topics. Probability and its Applications. Springer Verlag, 1995.

[9] D.W. Stroock, S.R.S. Varadhan: Multidimensional Diffusion Processes. Grundlehren des mathematischen Wissenschaften 233. Springer Verlag, 1979.

Laboratoire de Probabilités et Modìles Aléatoires (CNRS UMR 7599), Universités PARIS 6-PARIS 7, Boite Courrier 188, 4 Place Jussieu, 75252 PARis Cedex 05, and SAMOS-MATISSE, Université Paris 1, 90 Rue de Tolbiac, 75634 Paris Cedex 13

E-mail address: amil@ccr.jussieu.fr

Facultat de Matemàtiques, Universitat de Barcelona, Gran Via 585, E08007 BARCELONA

E-mail address: marta.sanz@ub.edu 